\title{
Theoretical Investigation of Enolborane Addition to $\alpha$-Heteroatom- Substituted Aldehydes. Relevance of the Cornforth and Polar Felkin- Anh Models for Asymmetric Induction
}

\author{
Victor J. Cee ${ }^{\dagger}$, Christopher J. Cramer,,$"$, and David A. Evans,,$+\uparrow$
}

Department of Chemistry and Chemical Biology, Harvard University, Cambridge, Massachusetts 02138 and Department of Chemistry and Supercomputing Institute, University of Minnesota, Minneapolis, Minnesota 55455

\section{Supporting Information}

Complete Reference 13: Frisch, M. J.; Trucks, G. W.; Schlegel, H. B.; Scuseria, G. E.; Robb, M. A.; Cheeseman, J. R.; Zakrzewski, V. G.; Montgomery Jr., J. A.; Stratmann, R. E.; Burant, J. C.; Dapprich, S.; Millam, J. M.; Daniels, A. D.; Kudin, K. N.; Strain, M. C.; Farkas, O.; Tomasi, J.; Barone, V.; Cossi, M.; Cammi, R.; Mennucci, B.; Pomelli, C.; Adamo, C.; Clifford, S.; Ochterski, J.; Petersson, G. A.; Ayala, P. Y.; Cui, Q.; Morokuma, K.; Salvador, P.; Dannenberg, J. J.; Malick, D. K.; Rabuck, A. D.; Raghavachari, K.; Foresman, J. B.; Cioslowski, J.; Ortiz, J. V.; Baboul, A. G.; Stefanov, B. B.; Liu, G.; Liashenko, A.; Piskorz, P.; Komaromi, I.; Gomperts, R.; Martin, R. L.; Fox, D. J.; Keith, T.; Al-Laham, M. A.; Peng, C. Y.; Nanayakkara, A.; Challacombe, M.; Gill, P. M. W.; Johnson, B.; Chen, W.; Wong, M. W.; Andres, J. L.; Gonzalez, C.; HeadGordon, M.; Replogle, E. S.; Pople, J. A. Gaussian 98 (Revision A.11), Gaussian, Inc.; Pittsburgh PA, 2001.

\section{Coordinates for acetaldehyde enolborane + formaldehyde}

$\begin{array}{cccr}\text { Reactant complex }(\mathrm{MP} 2 / 6-31 \mathrm{G}(\mathrm{d})) \\ \mathrm{B} & 0.559842 & 1.422939 & -0.071402 \\ \mathrm{O} & -0.509529 & 0.654123 & -0.532428 \\ \mathrm{C} & -1.425155 & 0.156611 & 0.368817 \\ \mathrm{C} & -2.350148 & -0.732983 & 0.001252 \\ \mathrm{C} & 1.692441 & -1.175470 & -0.138564 \\ \mathrm{O} & 1.958431 & -0.074461 & 0.339752 \\ \mathrm{H} & 0.545088 & 1.832582 & 1.053809 \\ \mathrm{H} & 1.229173 & 1.925491 & -0.921300 \\ \mathrm{H} & -1.336859 & 0.544339 & 1.382683 \\ \mathrm{H} & -3.067505 & -1.098333 & 0.724620 \\ \mathrm{H} & -2.416197 & -1.086100 & -1.021237 \\ \mathrm{H} & 2.384927 & -2.017249 & -0.005291 \\ \mathrm{H} & 0.768115 & -1.341668 & -0.703901\end{array}$

MP2 $=-292.874988$

Reactant complex (B3LYP/6-31G(d))

$\begin{array}{lrrr}\text { B } & 0.447423 & 1.465462 & -0.091892 \\ \text { O } & -0.555092 & 0.612591 & -0.508585 \\ \text { C } & -1.478950 & 0.108177 & 0.369459 \\ \text { C } & -2.406116 & -0.769976 & -0.006053\end{array}$

${ }^{\dagger}$ Harvard University

University of Minnesota 
Journal of the American Chemical Society

$\begin{array}{cccc}\mathrm{C} & 1.852851 & -1.130765 & -0.134937 \\ \mathrm{O} & 2.038920 & -0.029483 & 0.340466 \\ \mathrm{H} & 0.440555 & 1.877775 & 1.033170 \\ \mathrm{H} & 1.100631 & 1.962694 & -0.957877 \\ \mathrm{H} & -1.394566 & 0.485417 & 1.388505 \\ \mathrm{H} & -3.126004 & -1.140628 & 0.714433 \\ \mathrm{H} & -2.474144 & -1.120469 & -1.031101 \\ \mathrm{H} & 2.605097 & -1.931044 & -0.018598 \\ \mathrm{H} & 0.933981 & -1.370542 & -0.694936 \\ \mathrm{HF}=-293.8051441 & & \end{array}$

Transition state (MP2/6-31G(d))

$\begin{array}{cccc}\text { B } & -1.378663 & -0.687718 & 0.174702 \\ \text { O } & -0.073718 & -1.284721 & -0.315878 \\ \text { C } & 0.953265 & -0.755059 & 0.320060 \\ \text { C } & 1.706322 & 0.234583 & -0.246846 \\ \text { C } & -0.201483 & 1.426870 & 0.264017 \\ \text { O } & -1.111348 & 0.792086 & -0.350297 \\ \text { H } & -1.443982 & -0.634489 & 1.383655 \\ \text { H } & -2.306651 & -1.132733 & -0.429813 \\ \text { H } & 1.073917 & -1.005319 & 1.380685 \\ \text { H } & 2.561842 & 0.642225 & 0.282205 \\ \text { H } & 1.609987 & 0.435526 & -1.308390 \\ \text { H } & 0.22286 & 2.306091 & -0.221734 \\ \text { H } & -0.092172 & 1.330008 & 1.345905 \\ \text { MP2=-292.867615 } & & \end{array}$

Transition state (B3LYP/6-31G(d))
O
$-1.355382$
0.988409
1.133891
2.543679
0.108911
$-0.730357$
0.159187
$-0.300884$
$-1.292169$
$-0.729231$
0.289955
0.314691
$-0.248608$
1.434841
0.749367
0.258877
$-0.337435$
$-0.703071$
1.369384
$-1.216926$
$-0.979273$
$-0.461344$
1.373514
0.730872
0.285335
0.499822
2.330017
$-1.308804$
1.359370
$-0.239477$
$\mathrm{HF}=-293.800360$
1.342247

Product (MP2/6-31G(d))

$\begin{array}{lrrr}\text { B } & -1.696565 & -1.009150 & 0.180843 \\ \mathrm{O} & 1.131235 & -1.356008 & -0.152213 \\ \mathrm{C} & 1.547665 & -0.247642 & 0.164710 \\ \mathrm{C} & 0.867055 & 1.041647 & -0.233731 \\ \mathrm{C} & -0.562350 & 1.123190 & 0.308799 \\ \mathrm{O} & -1.391089 & 0.189848 & -0.380934 \\ \mathrm{H} & -1.412604 & -1.240255 & 1.321920 \\ \mathrm{H} & -2.311144 & -1.770219 & -0.500807\end{array}$


Journal of the American Chemical Society

$\begin{array}{rrrr}\mathrm{H} & 2.459471 & -0.158557 & 0.789154 \\ \mathrm{H} & 1.448833 & 1.896406 & 0.127992 \\ \mathrm{H} & 0.817864 & 1.093850 & -1.326488 \\ \mathrm{H} & -0.969960 & 2.120804 & 0.122695 \\ \mathrm{H} & -0.585014 & 0.929832 & 1.387828\end{array}$

MP2 $=-292.9153481$

Product (B3LYP/6-31G(d))

$\begin{array}{lrrr}\mathrm{B} & -2.066451 & -0.825864 & -0.054239 \\ \mathrm{O} & 1.232794 & -1.334868 & 0.250594 \\ \mathrm{C} & 1.635433 & -0.297133 & -0.225911 \\ \mathrm{C} & 0.909218 & 1.029252 & -0.135846 \\ \mathrm{C} & -0.476442 & 0.934586 & 0.489606 \\ \mathrm{O} & -1.356888 & 0.263902 & -0.413037 \\ \mathrm{H} & -2.024673 & -1.245699 & 1.068721 \\ \mathrm{H} & -2.747249 & -1.316269 & -0.906915 \\ \mathrm{H} & 2.608584 & -0.261016 & -0.765188 \\ \mathrm{H} & 1.544855 & 1.715411 & 0.445105 \\ \mathrm{H} & 0.846724 & 1.462035 & -1.144012 \\ \mathrm{H} & -0.876808 & 1.940166 & 0.659767 \\ \mathrm{H} & -0.435684 & 0.402191 & 1.446164\end{array}$

$\mathrm{HF}=-293.8455569$

\section{Coordinates for optimized fluoroacetaldehyde geometries}

B3LYP/6-31G(d) $\phi_{1}=0.0$

$\begin{array}{rrrrr}\text { C } & 0 & 0.771515 & 0.050018 & -0.000137 \\ \mathrm{O} & 0 & 1.359039 & -1.003067 & -0.000137 \\ \mathrm{C} & 0 & -0.736542 & 0.193024 & -0.000031 \\ \mathrm{~F} & 0 & -1.359024 & -1.028519 & 0.000031 \\ \mathrm{H} & 0 & 1.306305 & 1.028534 & -0.000137 \\ \mathrm{H} & 0 & -1.038467 & 0.770996 & 0.887451 \\ \mathrm{H} & 0 & -1.038620 & 0.770996 & -0.887436\end{array}$

$\mathrm{HF}=-253.0471834$

Zero-point correction $=\quad .048985$ (Hartree/Particle)

$\mathrm{MP} 2 / 6-31 \mathrm{G}(\mathrm{d}) \phi_{1}=0.0$
C $0 \quad 0.761703$
O 001.346725
0.066071
$-0.000031$
$-1.003418$
$-0.000244$
C $0 \quad-0.741486$
0.206207
0.000031
F $0 \quad-1.346725$
$-1.033920$
0.000122
$\mathrm{H} \quad 0 \quad 1.305634$
1.033936
$-0.000046$
$\mathrm{H} \quad 0 \quad-1.054214$
0.770050
$-1.054291$
0.769928
0.888397
$-0.888382$

MP2 $=-252.3510245$

Zero-point correction $=\quad .049930$ (Hartree/Particle)

B3LYP/6-311+G(3df,2p) $\phi_{1}=0.0$
C 0
0.781311
0.038422
$-0.000107$
O 001.374466
$-1.001114$
$-0.000122$
C $0 \quad-0.721146$
0.187317
$-0.000015$
F $0 \quad-1.374451$
$-1.016586$
0.000046
$\mathrm{H} \quad 0 \quad 1.310486$
1.016602
$-0.000137$ 
Journal of the American Chemical Society

$\begin{array}{rrrrr}\mathrm{H} & 0 & -1.017868 & 0.760529 & 0.886017 \\ \mathrm{H} & 0 & -1.017990 & 0.760513 & -0.886002\end{array}$

$\mathrm{HF}=-253.1541693$

Zero-point correction=

.048477 (Hartree/Particle)

$\mathrm{MP} 2 / 6-311+\mathrm{G}(3 \mathrm{df}, 2 \mathrm{p}) \phi_{1}=0.0$
C 0
0.769531
0.048782
$-0.000153$
O $0 \quad 1.363312$
$-1.000748$
$-0.000137$
C $0 \quad-0.730728$
0.189087
$-0.000031$
F $0 \quad-1.363297$
$-1.020981$
0.000046
$\mathrm{H} 0 \quad 1.300598$
1.020996
$-0.000107$
$\mathrm{H} 0 \quad-1.028854$
0.756088
0.886108
$\mathrm{H} \quad 0 \quad-1.029037$
0.756088
$-0.886108$

MP $2=-252.6572872$

B3LYP/6-31G(d) $\phi_{1}=180.0$
C 0
0.625122
$-0.218200$
0.000198
O 001.749283
0.225494
$-0.000122$
C $0 \quad-0.610611$
0.667206
0.000015
F $0 \quad-1.749283$
$-0.118164$
$-0.000076$
$\mathrm{H} 0 \quad 0.408295$
$-1.308807$
$-0.000092$
$-0.605331$
1.308792
0.890594
$\mathrm{H} 0 \quad-0.605103$
1.308746
$-0.890579$

$\mathrm{HF}=-253.0492089$

Zero-point correction=

.048711 (Hartree/Particle)

$\mathrm{MP} 2 / 6-31 \mathrm{G}(\mathrm{d}) \phi_{1}=180.0$
$\begin{array}{lll}C & 0 & 0.615067\end{array}$
O 001.751175
$-0.222809$
$-0.000015$
C $0 \quad-0.604400$
0.225983
$-0.000015$
F $0 \quad-1.751160$
0.673218
$-0.000015$
$\mathrm{H} 0 \quad 0.400894$
$-0.115738$
$-0.000015$
$-1.308716$
0.000015
$\mathrm{H} \quad 0 \quad-0.601044$
1.308685
$\mathrm{H} 0 \quad-0.601044$
1.308716
0.891113
$-0.891113$

MP $2=-252.3537697$

Zero-point correction=

.049722 (Hartree/Particle)

B3LYP/6-311+G(3df,2p) $\phi_{1}=180.0$
C 0
0.631348
$-0.212830$
$-0.000015$
$\begin{array}{lll}\mathrm{O} & 0 & 1.745468\end{array}$
0.232193
$-0.000015$
C $0 \quad-0.599930$
F $0 \quad-1.745453$
0.668350
$-0.000015$
$\mathrm{H} 0 \quad 0.424225$
$-0.110214$
$-0.000015$
$\mathrm{H} 0 \quad-0.602112$
$-1.301743$
0.000015
1.301712
0.889725
$\mathrm{H} \quad 0 \quad-0.602081$
1.301727
$-0.889725$

$\mathrm{HF}=-253.1561053$

Zero-point correction=

.048278 (Hartree/Particle)

$\mathrm{MP} 2 / 6-311+\mathrm{G}(3 \mathrm{df}, 2 \mathrm{p}) \phi_{1}=180.0$
C 0
0.621338
$-0.215012$
$-0.000076$
O $0 \quad 1.742722$
0.235733
$-0.000046$
C $0 \quad-0.602280$
0.670685
$-0.000046$
F $0 \quad-1.742722$
$-0.106232$
$-0.000046$ 
Journal of the American Chemical Society

$\begin{array}{rrrrr}\mathrm{H} & 0 & 0.417648 & -1.300247 & 0.000061 \\ \mathrm{H} & 0 & -0.599228 & 1.300140 & 0.889481 \\ \mathrm{H} & 0 & -0.599243 & 1.300232 & -0.889481\end{array}$

Coordinates for the optimized, unconstrained fluoroacetaldehyde transition state geometries

B3LYP/6-31G(d) $\phi_{1}=69.4$

$\begin{array}{rrrrr}\mathrm{C} & 0 & -0.716888 & -0.073380 & -0.412109 \\ \mathrm{O} & 0 & -1.459946 & -0.932785 & -0.002563 \\ \mathrm{C} & 0 & 0.716080 & 0.079361 & 0.108978 \\ \mathrm{~F} & 0 & 1.459930 & -1.003540 & -0.337448 \\ \mathrm{H} & 0 & -1.036057 & 0.637283 & -1.205307 \\ \mathrm{H} & 0 & 1.176331 & 1.003540 & -0.259384 \\ \mathrm{H} & 0 & 0.705536 & 0.077789 & 1.205322\end{array}$

$\mathrm{HF}=-253.0414822$

Zero-point correction $=\quad .048916$ (Hartree/Particle)

$\mathrm{MP} 2 / 6-31 \mathrm{G}(\mathrm{d}) \phi_{1}=68.0$

$\begin{array}{rrrrr}\mathrm{C} & 0 & -0.708313 & -0.053436 & -0.417328 \\ \mathrm{O} & 0 & -1.447418 & -0.936783 & -0.008881 \\ \mathrm{C} & 0 & 0.714035 & 0.092682 & 0.103226 \\ \mathrm{~F} & 0 & 1.447418 & -1.006042 & -0.343765 \\ \mathrm{H} & 0 & -1.041229 & 0.658463 & -1.196884 \\ \mathrm{H} & 0 & 1.188309 & 1.006042 & -0.267334 \\ \mathrm{H} & 0 & 0.704605 & 0.088500 & 1.196884\end{array}$

MP2 $=-252.3460162$

Zero-point correction $=\quad .049803$ (Hartree/Particle)

B3LYP/6-311+G(3df,2p) $\phi_{1}=68.0$

$\begin{array}{rrrrr}\mathrm{C} & 0 & -0.725830 & -0.069672 & -0.416245 \\ \mathrm{O} & 0 & -1.456177 & -0.932663 & -0.018173 \\ \mathrm{C} & 0 & 0.708191 & 0.085861 & 0.092834 \\ \mathrm{~F} & 0 & 1.456177 & -0.996368 & -0.347168 \\ \mathrm{H} & 0 & -1.060455 & 0.654922 & -1.184326 \\ \mathrm{H} & 0 & 1.172500 & 0.996384 & -0.288666 \\ \mathrm{H} & 0 & 0.709564 & 0.085068 & 1.184341\end{array}$

$\mathrm{HF}=-253.1482462$

Zero-point correction=

.048409 (Hartree/Particle)

$\mathrm{MP} 2 / 6-311+\mathrm{G}(3 \mathrm{df}, 2 \mathrm{p}) \phi_{1}=67.0$
C 0
$-0.715500$
$-0.052887$
$-0.420746$
O $00-1.443680$
$-0.933380$
$-0.026215$
C $0 \quad 0.712708$
0.090439
0.086090
F $0 \quad 1.443680$
$-0.996094$
$-0.355240$
$-1.058807$
0.675186
$-1.175613$
$\mathrm{H} \quad 0 \quad 1.183624$
0.996094
$-0.293610$
$\mathrm{H} \quad 0 \quad 0.712341$
0.087494
1.175629

MP2 $=-252.6517758$

Coordinates for optimized, unconstrained chloroacetaldehyde geometries

B3LYP/6-31G(d) $\phi_{1}=0.0$ 
Journal of the American Chemical Society
C 0
0.958969
$-0.032486$
0.000000
O 0
1.233459
$-1.204529$
0.000000
C 0
$-0.436218$
0.565521
0.000000
Cl $0 \quad-1.747849$
$-0.649536$
0.000000
$\mathrm{H} \quad 0$
1.747864
0.754593
$-0.000061$
H $0 \quad-0.547562$
1.204529
1.204529
0.883514

$\begin{array}{lr}\mathrm{H} O & -0.547562 \\ \mathrm{HF}=-613.4178003\end{array}$

Zero-point correction=

.047477 (Hartree/Particle)

MP2/6-31G(d) $\phi_{1}=0.0$
$\begin{array}{lll}\text { C } 0 & -0.938446\end{array}$
$-0.019089$
0.000000
$\begin{array}{lll}0 & 0 & -1.210571\end{array}$
$-1.205750$
0.000000
C 0
0.456406
0.569077
0.000000
$\mathrm{Cl} 0$
1.730225
$-0.652664$
0.000000
$\mathrm{H} 0 \quad-1.730240$
0.758011
$\mathrm{H} \quad \mathrm{O}$
0.568253
1.205765
$\mathrm{H} O$
0.568253
1.205765
0.000000
$-0.883347$
0.883347

MP2 $=-612 \cdot 3685762$

Zero-point correction $=\quad .048539$ (Hartree/Particle)

B3LYP/6-311+G(3df,2p) $\phi_{1}=0.0$
C 0
0.958344
$-0.031113$
0.000000
O $0 \quad 1.239960$
$-1.193008$
0.000000
C $0 \quad-0.433563$
0.556702
0.000000
Cl $0 \quad-1.743011$
$-0.643936$
0.000000
$\mathrm{H} \quad 0$
1.743027
0.756088
0.000000
H $0 \quad-0.542038$
1.193008
0.880005
H $0 \quad-0.542053$
1.193008
$-0.879990$

$\mathrm{HF}=-613.51171$

Zero-point correction=

.046919 (Hartree/Particle)

MP2/6-311+G(3df,2p) $\phi_{1}=0.0$
C 0
$-0.937042$
$-0.022476$
$-0.000076$
$\begin{array}{lll}\mathrm{O} & 0 & -1.207428\end{array}$
$-1.196716$
$-0.000031$
C $0 \quad 0.454880$
0.561707
$-0.000031$
$\mathrm{Cl} 0 \quad 1.726913$
$-0.647720$
$-0.000031$
H $0 \quad-1.726913$
0.753235
0.000031
H $0 \quad 0.561737$
1.196732
$-0.880600$
H $0 \quad 0.561691$
1.196686
0.880600
MP2 $=-612.6402896$

B3LYP/6-31G(d) $\phi_{1}=152.7$
C 0
0.809784
$-0.350067$
$-0.014145$
$\begin{array}{ll}0 & 0\end{array}$
1.927094
$-0.061234$
$-0.368347$
C 0
$-0.332794$
0.654877
$-0.057465$
Cl $0 \quad-1.927094$
$-0.167923$
$-0.250229$
$\mathrm{H} \quad 0$
0.551117
$-1.352219$
0.385681
$\mathrm{H} \quad 0 \quad-0.362808$
1.213623
0.883774
$\mathrm{H} \quad 0 \quad-0.187744$
1.352234
$-0.883774$

$\mathrm{HF}=-613.4191655$

Zero-point correction=

.047393 (Hartree/Particle) 


\begin{tabular}{|c|c|c|c|}
\hline \multicolumn{4}{|c|}{$\mathrm{MP} 2 / 6-31 \mathrm{G}(\mathrm{d}) \phi_{1}=162.6$} \\
\hline C 0 & 0.781982 & -0.325317 & -0.025406 \\
\hline $\mathrm{O}$ & 1.933487 & 0.007523 & -0.252472 \\
\hline C 0 & -0.350433 & 0.685059 & -0.043747 \\
\hline $\mathrm{Cl} 0$ & -1.933487 & -0.111343 & -0.178604 \\
\hline $\mathrm{H} \quad 0$ & 0.504471 & -1.366364 & 0.226151 \\
\hline 0 & -0.331604 & 1.262421 & 0.884369 \\
\hline 0 & -0.213181 & 1.366364 & -0.884369 \\
\hline
\end{tabular}

MP $2=-612.3698918$

Zero-point correction $=\quad .048357$ (Hartree/Particle)

B3LYP/6-311+G(3df,2p) $\phi_{1}=149.7$
C 0
0.807343
$-0.353012$
$-0.001968$
O $0 \quad 1.909561$
$-0.087250$
$-0.392609$
C $0 \quad-0.332428$
$0.643570-0.058090$
$\begin{array}{lll}\mathrm{Cl} & 0 & -1.909546 \\ \mathrm{H} & 0 & 0.560379\end{array}$
$-0.188614$
$-0.261917$
0.560379
$-1.336838$
0.439300
$\mathrm{H} 0 \quad-0.374710$
1.195007
0.881287
$\mathrm{H} 0 \quad-0.189133$
1.336853
$-0.881271$

$\mathrm{HF}=-613.5134005$

Zero-point correction $=\quad .046903$ (Hartree/Particle)

$\mathrm{MP} 2 / 6-311+\mathrm{G}(3 \mathrm{df}, 2 \mathrm{p}) \phi_{1}=145.6$
C 0
0.790741
$-0.361404$
0.023071
O 0
1.888260
$-0.121277$
$-0.420792$
C $0 \quad-0.348419$
0.629822
$-0.054413$
$\mathrm{Cl} 0 \quad-1.888260$
$-0.218094$
$-0.273788$
$\mathrm{H} \quad 0$
0.557556
$-1.315735$
0.526276
$\mathrm{H} 0 \quad-0.403030$
1.186325
0.881104
$\mathrm{H} \quad 0 \quad-0.189865$
1.315735
$-0.881104$
MP2 $=-612.6416707$

\section{Coordinates for optimized, unconstrained chloroacetaldehyde transition state geometries}

B3LYP/6-31G(d) $\phi_{1}=60.4$
C 0
$-1.086380$
$-0.240570$
$-0.395721$
$\begin{array}{lll}\mathrm{O} & 0 & -1.573471\end{array}$
$-1.258255$
0.029419
C $0 \quad 0.246400$
0.326630
0.093460
Cl $0 \quad 1.598297$
$-0.834824$
$-0.198792$
$\begin{array}{rrr}\mathrm{H} & 0 & -1.598297 \\ \mathrm{H} & 0 & 0.492798\end{array}$
0.368088
$-1.171906$
$\mathrm{H} \quad 0 \quad 0.492798$
1.258255
$-0.418732$
$\begin{array}{lll}\mathrm{H} & 0 & 0.194092\end{array}$
0.498474
1.171921

$\mathrm{HF}=-613.4141545$

Zero-point correction $=\quad .047430$ (Hartree/Particle)

$\mathrm{MP} 2 / 6-31 \mathrm{G}(\mathrm{d}) \phi_{1}=61.0$

$\begin{array}{llccc}\text { C } & 0 & -1.066818 & -0.230026 & -0.399277 \\ \text { O } & 0 & -1.560760 & -1.257782 & 0.033798 \\ \text { C } & 0 & 0.259689 & 0.320847 & 0.100754 \\ \mathrm{Cl} & 0 & 1.575974 & -0.839127 & -0.198196 \\ \mathrm{H} & 0 & -1.575974 & 0.369064 & -1.178696 \\ \mathrm{H} & 0 & 0.507797 & 1.257797 & -0.399628 \\ \mathrm{H} & 0 & 0.194382 & 0.484604 & 1.178696\end{array}$


MP 2 $=-612.3651404$

Zero-point correction $=\quad .048461$ (Hartree/Particle)

B3LYP/6-311+G(3df,2p) $\phi_{1}=58.5$
$\begin{array}{lll}C & 0 & -1.069092\end{array}$
$-0.232224$
$-0.397598$
O $00-1.542100$
$-1.253159$
0.011536
C $0 \quad 0.263824$
0.333221
0.074234
$\mathrm{Cl} 0$
1.602661
$-0.826050$
$-0.210403$
$\begin{array}{rrr}\mathrm{H} & 0 & -1.602676 \\ \mathrm{H} & 0 & 0.508545\end{array}$
0.386581
$-1.146240$
$\begin{array}{lll}\mathrm{H} & 0 & 0.508545 \\ \mathrm{H} & 0 & 0.215973\end{array}$
1.253159
$-0.449219$
0.517517
1.146240

$\mathrm{HF}=-613.5080698$

Zero-point correction=

.046894 (Hartree/Particle)

$\mathrm{MP} 2 / 6-311+\mathrm{G}(3 \mathrm{df}, 2 \mathrm{p}) \phi_{1}=58.3$
C $0 \quad-1.048111$
$\begin{array}{lll}\mathrm{O} & 0 & -1.516647\end{array}$
$-0.223770$
$-1.255585$
$-0.400116$
0.015213
C $0 \quad 0.280945$
0.333939
0.078171
Cl $0 \quad 1.582855$
$-0.831177$
$-0.210846$
$\mathrm{H} 0 \quad-1.582855$
0.385147
$-1.150208$
$\mathrm{H} \quad 0 \quad 0.529449$
1.255585
$-0.440536$
$\mathrm{H} \quad 0 \quad 0.226028$
0.514267
1.150208
MP2 $=-612.6366493$

Coordinates for optimized, unconstrained geometries of 2-fluoropropanal (1).

$\phi_{1}=61.8(\mathrm{TS})$

$\begin{array}{rrrr}\mathrm{H} & 0.803918 & -1.472420 & -0.798119 \\ \mathrm{C} & 0.918288 & -0.561320 & -0.167512 \\ \mathrm{O} & 2.005517 & -0.097973 & 0.076630 \\ \mathrm{C} & -0.386672 & 0.066310 & 0.350323 \\ \mathrm{H} & -0.299472 & 0.176975 & 1.439161 \\ \mathrm{C} & -1.623254 & -0.721336 & -0.033575 \\ \mathrm{~F} & -0.465601 & 1.348938 & -0.195331 \\ \mathrm{H} & -1.590615 & -1.726233 & 0.403616 \\ \mathrm{H} & -2.523686 & -0.216911 & 0.327439 \\ \mathrm{H} & -1.694038 & -0.819992 & -1.122576\end{array}$

$\mathrm{HF}=-292.3649611$

$\phi_{1}=189.0$

$\begin{array}{ccrr}\mathrm{H} & -0.971832 & -1.545623 & -0.547696 \\ \mathrm{C} & -0.956458 & -0.526638 & -0.102844 \\ \mathrm{O} & -1.953611 & 0.147649 & 0.011906 \\ \mathrm{C} & 0.422266 & -0.067363 & 0.359020 \\ \mathrm{H} & 0.438803 & -0.106415 & 1.458185 \\ \mathrm{C} & 0.771792 & 1.328214 & -0.132016 \\ \mathrm{~F} & 1.352821 & -0.994663 & -0.109625 \\ \mathrm{H} & 0.000561 & 2.031546 & 0.194405 \\ \mathrm{H} & 1.740562 & 1.641388 & 0.267010 \\ \mathrm{H} & 0.819806 & 1.344603 & -1.225477\end{array}$

$\mathrm{HF}=-292.3719713$ 
$\phi_{1}=292.8(\mathrm{TS})$

$\begin{array}{rrrr}\mathrm{H} & -1.553608 & -0.249630 & 1.455327 \\ \mathrm{C} & -1.055299 & -0.035808 & 0.484484 \\ \mathrm{O} & -1.696379 & 0.250517 & -0.498628 \\ \mathrm{C} & 0.479273 & -0.140583 & 0.461733 \\ \mathrm{H} & 0.850513 & -0.356251 & 1.471656 \\ \mathrm{C} & 1.126378 & 1.101627 & -0.122557 \\ \mathrm{~F} & 0.790500 & -1.241386 & -0.341559 \\ \mathrm{H} & 0.953738 & 1.971769 & 0.520814 \\ \mathrm{H} & 2.205044 & 0.946554 & -0.218477 \\ \mathrm{H} & 0.698728 & 1.304482 & -1.108230 \\ \mathrm{HF}=-292 & 3652966 & & \end{array}$

$\phi_{1}=356.4$

\section{Coordinates for optimized, unconstrained geometries of $1 \cdot \mathbf{B H}_{3}$}

\begin{tabular}{|c|c|c|c|}
\hline \multicolumn{4}{|c|}{$\phi_{1}=63.0(\mathrm{TS})$} \\
\hline $\mathrm{H}$ & 0.499449 & -1.314052 & -0.675233 \\
\hline $\mathrm{C}$ & 0.378230 & -0.407303 & -0.064970 \\
\hline 0 & 1.374427 & 0.226925 & 0.269674 \\
\hline $\mathrm{C}$ & -1.007029 & 0.065583 & 0.349150 \\
\hline $\mathrm{H}$ & -1.001657 & 0.218311 & 1.436264 \\
\hline $\mathrm{C}$ & -2.105686 & -0.887958 & -0.077020 \\
\hline $\mathrm{F}$ & -1.201418 & 1.307514 & -0.251028 \\
\hline $\mathrm{H}$ & -1.971917 & -1.867586 & 0.395968 \\
\hline $\mathrm{H}$ & -3.078955 & -0.490234 & 0.221647 \\
\hline $\mathrm{H}$ & -2.102371 & -1.019797 & -1.164168 \\
\hline B & 2.887011 & -0.194230 & -0.146345 \\
\hline $\mathrm{H}$ & 3.270868 & 0.774207 & -0.755118 \\
\hline $\mathrm{H}$ & 2.755124 & -1.207856 & -0.798404 \\
\hline $\mathrm{H}$ & 3.418660 & -0.326808 & 0.929669 \\
\hline \multicolumn{4}{|c|}{$\mathrm{HF}=-319.0048954$} \\
\hline \multicolumn{4}{|c|}{$\phi_{1}=181.7$} \\
\hline $\mathrm{H}$ & -0.550873 & -1.388136 & -0.581176 \\
\hline $\mathrm{C}$ & -0.383454 & -0.446127 & -0.039977 \\
\hline 0 & -1.329614 & 0.285932 & 0.240123 \\
\hline $\mathrm{C}$ & 1.023503 & -0.075136 & 0.353626 \\
\hline $\mathrm{H}$ & 1.083321 & -0.087377 & 1.451590 \\
\hline $\mathrm{C}$ & 1.448136 & 1.285233 & -0.189139 \\
\hline
\end{tabular}


Journal of the American Chemical Society

$\begin{array}{llll}\mathrm{F} & 1.859857 & -1.071382 & -0.134785 \\ \mathrm{H} & 0.774952 & 2.062385 & 0.183074 \\ \mathrm{H} & 2.467958 & 1.504053 & 0.137832 \\ \mathrm{H} & 1.420530 & 1.285368 & -1.282800 \\ \mathrm{~B} & -2.880440 & -0.058300 & -0.148419 \\ \mathrm{H} & -3.417697 & -0.079206 & 0.931799 \\ \mathrm{H} & -2.815640 & -1.122797 & -0.724181 \\ \mathrm{H} & -3.191261 & 0.888376 & -0.829020\end{array}$

$\phi_{1}=288.6(\mathrm{TS})$

$\begin{array}{crrr}\mathrm{H} & -0.824548 & -0.556925 & 1.579053 \\ \mathrm{C} & -0.398105 & -0.195391 & 0.633195 \\ \mathrm{O} & -1.157392 & 0.138052 & -0.272833 \\ \mathrm{C} & 1.109162 & -0.132872 & 0.429400 \\ \mathrm{H} & 1.604836 & -0.352071 & 1.381941 \\ \mathrm{C} & 1.571194 & 1.191032 & -0.152406 \\ \mathrm{~F} & 1.417217 & -1.168634 & -0.453929 \\ \mathrm{H} & 1.376684 & 2.013306 & 0.544657 \\ \mathrm{H} & 2.646800 & 1.142736 & -0.343930 \\ \mathrm{H} & 1.044901 & 1.391866 & -1.089093 \\ \mathrm{~B} & -2.776829 & 0.076143 & -0.159937 \\ \mathrm{H} & -3.089105 & 1.224872 & -0.362946 \\ \mathrm{H} & -2.991019 & -0.339273 & 0.958542 \\ \mathrm{H} & -3.073729 & -0.668547 & -1.061637 \\ \mathrm{HF}=-319.0051303 & & \end{array}$

$\phi_{1}=355.6$

$\begin{array}{cccc}\mathrm{H} & -0.629147 & -1.355136 & -0.860688 \\ \mathrm{C} & -0.378585 & -0.389085 & -0.398403 \\ \mathrm{O} & -1.262525 & 0.283094 & 0.122108 \\ \mathrm{C} & 1.056636 & 0.054135 & -0.417278 \\ \mathrm{H} & 1.371449 & 0.097335 & -1.472409 \\ \mathrm{C} & 1.944008 & -0.928120 & 0.350387 \\ \mathrm{~F} & 1.167219 & 1.314267 & 0.126493 \\ \mathrm{H} & 1.884188 & -1.933960 & -0.077698 \\ \mathrm{H} & 2.981025 & -0.586827 & 0.296378 \\ \mathrm{H} & 1.640474 & -0.969215 & 1.400567 \\ \mathrm{~B} & -2.824143 & -0.194155 & 0.168662 \\ \mathrm{H} & -3.048011 & -0.233331 & 1.353533 \\ \mathrm{H} & -2.827151 & -1.265934 & -0.400511 \\ \mathrm{H} & -3.389243 & 0.703108 & -0.406022\end{array}$

$\mathrm{HF}=-319.0095865$

Coordinates for optimized, unconstrained geometries of 2-chloropropanal (2)

$\begin{array}{crrr}\phi_{1}=51.9(\mathrm{TS}) & & & \\ \mathrm{H} & -1.616093 & 1.306136 & -0.718007 \\ \mathrm{C} & -1.314901 & 0.396803 & -0.150286 \\ \mathrm{O} & -2.084293 & -0.512186 & 0.031880 \\ \mathrm{C} & 0.122438 & 0.440552 & 0.389279 \\ \mathrm{H} & 0.063603 & 0.410970 & 1.482254 \\ \mathrm{C} & 0.891803 & 1.663962 & -0.085827\end{array}$


Journal of the American Chemical Society

$\begin{array}{rrrr}\mathrm{Cl} & 0.987537 & -1.091790 & -0.078303 \\ \mathrm{H} & 0.368725 & 2.574903 & 0.231925 \\ \mathrm{H} & 1.898341 & 1.681152 & 0.338316 \\ \mathrm{H} & 0.975599 & 1.676864 & -1.177379\end{array}$

$\mathrm{HF}=-652.7326096$

$\phi_{1}=135.6$

$\begin{array}{rrrr}\mathrm{H} & 0.982429 & -0.678716 & 1.410684 \\ \mathrm{C} & 1.183212 & -0.401744 & 0.354043 \\ \mathrm{O} & 2.248136 & -0.608567 & -0.176869 \\ \mathrm{C} & 0.030743 & 0.296851 & -0.355575 \\ \mathrm{H} & 0.188666 & 0.228692 & -1.433006 \\ \mathrm{C} & -0.105920 & 1.745793 & 0.104926 \\ \mathrm{Cl} & -1.494096 & -0.638282 & 0.010179 \\ \mathrm{H} & 0.804127 & 2.296280 & -0.160606 \\ \mathrm{H} & -0.959388 & 2.223074 & -0.384147 \\ \mathrm{H} & -0.249501 & 1.804603 & 1.188610\end{array}$

$\phi_{1}=170.0(\mathrm{TS})$

$\begin{array}{rrrr}\mathrm{H} & -0.767604 & -1.481660 & -0.897819 \\ \mathrm{C} & -1.108692 & -0.686622 & -0.203237 \\ \mathrm{O} & -2.271891 & -0.529599 & 0.081937 \\ \mathrm{C} & -0.024249 & 0.229447 & 0.366672 \\ \mathrm{H} & -0.107371 & 0.215257 & 1.456686 \\ \mathrm{C} & -0.168934 & 1.657047 & -0.159482 \\ \mathrm{Cl} & 1.617615 & -0.454100 & -0.022609 \\ \mathrm{H} & -1.170395 & 2.020542 & 0.093554 \\ \mathrm{H} & 0.576565 & 2.311853 & 0.298767 \\ \mathrm{H} & -0.044270 & 1.691266 & -1.246051\end{array}$

$\mathrm{HF}=-652.7373758$

$\phi_{1}=224.8$

$\begin{array}{cccc}\mathrm{H} & 0.054495 & 0.721664 & 0.239915 \\ \mathrm{C} & 0.039696 & 0.011206 & 1.091591 \\ \mathrm{O} & 1.013044 & -0.630735 & 1.407471 \\ \mathrm{C} & -1.291470 & -0.074992 & 1.833678 \\ \mathrm{H} & -1.467079 & 0.888081 & 2.327269 \\ \mathrm{C} & -1.370074 & -1.227373 & 2.818106 \\ \mathrm{Cl} & -2.600301 & -0.182396 & 0.558322 \\ \mathrm{H} & -0.570468 & -1.120023 & 3.558236 \\ \mathrm{H} & -2.334216 & -1.231433 & 3.332221 \\ \mathrm{H} & -1.232428 & -2.184001 & 2.307690 \\ \mathrm{HF}=-652.7385092 & & \end{array}$

$\begin{array}{ccrrr}\phi_{1}=305.0(\mathrm{TS}) & & & \\ \mathrm{H} & 1 & 0.112640 & -1.771378 & 1.567291 \\ \mathrm{C} & 2 & -0.288757 & -0.850647 & 1.089127 \\ \mathrm{O} & 3 & -1.361725 & -0.397568 & 1.401733 \\ \mathrm{C} & 4 & 0.653000 & -0.198074 & 0.071442 \\ \mathrm{H} & 5 & 1.468826 & -0.886597 & -0.161087 \\ \mathrm{C} & 6 & 1.185898 & 1.128967 & 0.608292\end{array}$




$\begin{array}{rrrrr}\mathrm{Cl} & 7 & -0.231949 & 0.058731 & -1.498337 \\ \mathrm{H} & 8 & 1.751236 & 0.957581 & 1.532944 \\ \mathrm{H} & 9 & 1.850128 & 1.598846 & -0.121597 \\ \mathrm{H} & 10 & 0.353790 & 1.803055 & 0.827789\end{array}$
$\mathrm{HF}=-652.7329048$

$\phi_{1}=357.0$

$\begin{array}{ccrr}\mathrm{H} & -2.024584 & 1.031379 & 0.536578 \\ \mathrm{C} & -1.380183 & 0.185414 & 0.201706 \\ \mathrm{O} & -1.851402 & -0.807440 & -0.289709 \\ \mathrm{C} & 0.097233 & 0.472010 & 0.441800 \\ \mathrm{H} & 0.229131 & 0.603486 & 1.522872 \\ \mathrm{C} & 0.529554 & 1.748014 & -0.287225 \\ \mathrm{Cl} & 1.130571 & -0.930046 & -0.028552 \\ \mathrm{H} & -0.103788 & 2.589178 & 0.019918 \\ \mathrm{H} & 1.566446 & 1.991284 & -0.043423 \\ \mathrm{H} & 0.444681 & 1.622343 & -1.370563\end{array}$

$\mathrm{HF}=-652.7350216$

Coordinates for optimized, unconstrained geometries of $\mathbf{2} \cdot \mathbf{B H}$

\begin{tabular}{crrr}
\multicolumn{4}{l}{$\phi_{1}=50.3(\mathrm{TS})$} \\
$\mathrm{H}$ & -0.994530 & 1.518894 & -0.549269 \\
$\mathrm{C}$ & -0.700166 & 0.598440 & -0.023642 \\
$\mathrm{O}$ & -1.556790 & -0.241515 & 0.229872 \\
$\mathrm{C}$ & 0.743173 & 0.435193 & 0.399282 \\
$\mathrm{H}$ & 0.762439 & 0.425190 & 1.495081 \\
$\mathrm{C}$ & 1.634196 & 1.541532 & -0.151470 \\
$\mathrm{Cl}$ & 1.345171 & -1.203666 & -0.104234 \\
$\mathrm{H}$ & 1.268867 & 2.517487 & 0.191094 \\
$\mathrm{H}$ & 2.659595 & 1.415337 & 0.202163 \\
$\mathrm{H}$ & 1.641131 & 1.534352 & -1.245667 \\
$\mathrm{~B}$ & -3.126900 & -0.065203 & -0.166085 \\
$\mathrm{H}$ & -3.330036 & -1.024952 & -0.867612 \\
$\mathrm{H}$ & -3.181794 & 1.013010 & -0.718522 \\
$\mathrm{H}$ & -3.667985 & -0.129857 & 0.911144
\end{tabular}

$\mathrm{HF}=-679.3739535$

$\phi_{1}=125.7$

$\begin{array}{cccc}\mathrm{H} & 0.743034 & -0.170202 & 1.323112 \\ \mathrm{C} & 0.695174 & 0.038643 & 0.246029 \\ \mathrm{O} & 1.719242 & 0.006343 & -0.432461 \\ \mathrm{C} & -0.627062 & 0.388408 & -0.372847 \\ \mathrm{H} & -0.525928 & 0.392591 & -1.459038 \\ \mathrm{C} & -1.162907 & 1.717252 & 0.156559 \\ \mathrm{Cl} & -1.755245 & -0.980862 & 0.047310 \\ \mathrm{H} & -0.483639 & 2.527479 & -0.132327 \\ \mathrm{H} & -2.147664 & 1.916856 & -0.273913 \\ \mathrm{H} & -1.254923 & 1.702854 & 1.246601 \\ \mathrm{~B} & 3.195733 & -0.308201 & 0.187568 \\ \mathrm{H} & 3.539746 & -1.277553 & -0.442522 \\ \mathrm{H} & 2.997837 & -0.487543 & 1.369682\end{array}$


Journal of the American Chemical Society

$\begin{array}{cccc}\mathrm{H} & 3.806879 & 0.694612 & -0.092462 \\ \mathrm{HF}=-679.3769462 & & \\ \phi_{1}=177.5 & & & \\ \mathrm{H} S & & & \\ \mathrm{C} & -0.663699 & -1.317245 & -0.558009 \\ \mathrm{O} & -0.679349 & -0.357688 & -0.024590 \\ \mathrm{C} & -1.750245 & 0.183011 & 0.238157 \\ \mathrm{H} & 0.608969 & 0.312782 & 0.389819 \\ \mathrm{C} & 0.616863 & 0.373839 & 1.483060 \\ \mathrm{Cl} & 0.748930 & 1.712723 & -0.215748 \\ \mathrm{H} & 2.001860 & -0.754033 & -0.067107 \\ \mathrm{H} & -0.110121 & 2.319190 & 0.087692 \\ \mathrm{H} & 1.666303 & 2.182652 & 0.145953 \\ \mathrm{~B} & 0.783532 & 1.665929 & -1.307621 \\ \mathrm{H} & -3.207471 & -0.449704 & -0.166266 \\ \mathrm{H} & -3.741065 & -0.566169 & 0.909308 \\ \mathrm{H} & -2.935800 & -1.483689 & -0.736012 \\ \mathrm{HF}=-679.3755897 & 0.421590 & -0.854356\end{array}$

$\phi_{1}=233.5$

\begin{tabular}{|c|c|c|c|}
\hline $\mathrm{H}$ & -0.762057 & -1.332991 & 0.837933 \\
\hline $\mathrm{C}$ & -0.661336 & -0.321830 & 0.423625 \\
\hline 0 & -1.629880 & 0.222419 & -0.100706 \\
\hline $\mathrm{C}$ & 0.669681 & 0.374423 & 0.512026 \\
\hline $\mathrm{H}$ & 0.943839 & 0.443795 & 1.570991 \\
\hline $\mathrm{C}$ & 0.728875 & 1.727529 & -0.174419 \\
\hline $\mathrm{Cl}$ & 1.871632 & -0.806650 & -0.202086 \\
\hline $\mathrm{H}$ & 0.002981 & 2.402855 & 0.290575 \\
\hline $\mathrm{H}$ & 1.727800 & 2.157331 & -0.071280 \\
\hline $\mathrm{H}$ & 0.485017 & 1.639016 & -1.235643 \\
\hline B & -3.106016 & -0.468866 & -0.206201 \\
\hline $\mathrm{H}$ & -2.972529 & -1.552755 & 0.318346 \\
\hline $\mathrm{H}$ & -3.797991 & 0.319750 & 0.390736 \\
\hline $\mathrm{H}$ & -3.299003 & -0.479704 & -1.396948 \\
\hline & 9.377144 & & \\
\hline
\end{tabular}

$\phi_{1}=305.3(\mathrm{TS})$

$\begin{array}{rrrr}\mathrm{H} & -1.208849 & 0.494215 & 1.610118 \\ \mathrm{C} & -0.720961 & 0.255145 & 0.654236 \\ \mathrm{O} & -1.410045 & -0.131712 & -0.284600 \\ \mathrm{C} & 0.766848 & 0.472997 & 0.513395 \\ \mathrm{H} & 1.189966 & 0.638824 & 1.506112 \\ \mathrm{C} & 1.055921 & 1.660061 & -0.410148 \\ \mathrm{Cl} & 1.548997 & -1.041399 & -0.113312 \\ \mathrm{H} & 0.602440 & 2.572260 & -0.004223 \\ \mathrm{H} & 2.135100 & 1.813346 & -0.487568 \\ \mathrm{H} & 0.643546 & 1.474563 & -1.404969 \\ \mathrm{~B} & -3.026235 & -0.308694 & -0.195604 \\ \mathrm{H} & -3.410365 & 0.434382 & -1.066282 \\ \mathrm{H} & -3.305522 & 0.020822 & 0.937645 \\ \mathrm{H} & -3.178579 & -1.476672 & -0.454603\end{array}$




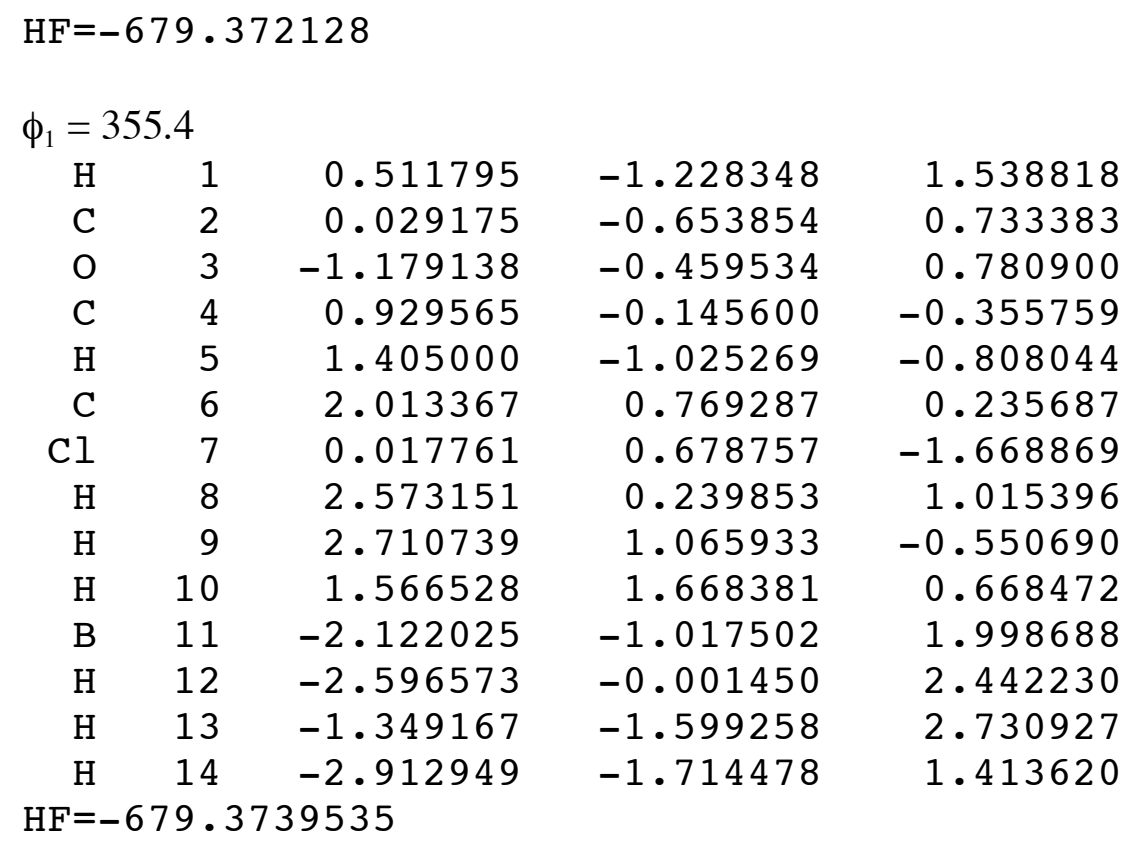

Coordinates for transition state geometries for the reaction of acetaldehyde enolborane (7) with 2fluoropropanal (1)

\begin{tabular}{llrrr}
\multicolumn{1}{l}{ 1A } & & & \\
C & 0 & -2.020065 & -0.215851 & 1.129715 \\
C & 0 & -1.650070 & -0.295776 & -0.190445 \\
C & 0 & 0.444427 & 0.053589 & 0.614502 \\
O & 0 & 0.551834 & -0.871323 & 1.486877 \\
B & 0 & -0.398163 & -0.839386 & 2.709000 \\
O & 0 & -1.720413 & -1.147522 & 1.989136 \\
C & 0 & 1.248383 & -0.101151 & -0.665192 \\
F & 0 & 1.008942 & -1.349213 & -1.225616 \\
C & 0 & 1.031387 & 0.984314 & -1.704849 \\
H & 0 & -2.418884 & 0.727844 & 1.526703 \\
H & 0 & -1.941238 & 0.496643 & -0.873657 \\
H & 0 & -1.332611 & -1.242203 & -0.613342 \\
H & 0 & 0.259048 & 1.079330 & 0.952438 \\
H & 0 & -0.116714 & -1.738861 & 3.444275 \\
H & 0 & -0.400604 & 0.286896 & 3.163391 \\
H & 0 & 2.300522 & -0.096054 & -0.338654 \\
H & 0 & 1.717392 & 0.828430 & -2.542007 \\
H & 0 & 0.009094 & 0.966034 & -2.090683 \\
H & 0 & 1.231064 & 1.973740 & -1.278854
\end{tabular}

$\mathrm{HF}=-471.6639768$

Zero-point correction $=\quad .150841$ (Hartree/Particle)

$1 B$

$\begin{array}{rrrrr}\text { C } & 0 & -2.439407 & -0.112381 & 0.997437 \\ \text { C } & 0 & -2.067902 & -0.198761 & -0.320679 \\ \text { C } & 0 & 0.000656 & 0.104248 & 0.528503 \\ \text { O } & 0 & 0.100693 & -0.821396 & 1.404022 \\ \text { B } & 0 & -0.885406 & -0.792084 & 2.608902 \\ \text { O } & 0 & -2.187393 & -1.069351 & 1.847641\end{array}$


Journal of the American Chemical Society

$\begin{array}{rrrrr}\text { C } & 0 & 0.799606 & -0.062119 & -0.746689 \\ \text { F } & 0 & 0.329056 & 0.859985 & -1.680130 \\ \text { C } & 0 & 2.278946 & 0.205841 & -0.488770 \\ \text { H } & 0 & -2.773010 & 0.850555 & 1.406570 \\ \text { H } & 0 & -2.277054 & 0.613632 & -1.008179 \\ \text { H } & 0 & -1.817169 & -1.167435 & -0.740967 \\ \text { H } & 0 & -0.179977 & 1.135529 & 0.850540 \\ \text { H } & 0 & -0.632202 & -1.707626 & 3.334991 \\ \text { H } & 0 & -0.879471 & 0.326569 & 3.079300 \\ \text { H } & 0 & 0.653610 & -1.070297 & -1.147491 \\ \text { H } & 0 & 2.831223 & 0.126282 & -1.430054 \\ \text { H } & 0 & 2.424240 & 1.212509 & -0.083008 \\ \text { H } & 0 & 2.675644 & -0.523987 & 0.222763 \\ \text { H } & & -6680987 & & \end{array}$

$\mathrm{HF}=-471.6680987$

Zero-point correction=

.150921 (Hartree/Particle)

1C

$\begin{array}{rrrrr}\text { C } & 0 & -2.346588 & 0.395935 & 0.863922 \\ \text { C } & 0 & -1.956055 & 0.301788 & -0.453339 \\ \text { C } & 0 & 0.082413 & 0.637238 & 0.355347 \\ \text { O } & 0 & 0.201813 & -0.282120 & 1.238007 \\ \text { B } & 0 & -0.743286 & -0.247681 & 2.457535 \\ \text { O } & 0 & -2.076492 & -0.540573 & 1.721634 \\ \text { C } & 0 & 0.888596 & 0.518753 & -0.932053 \\ \text { F } & 0 & 2.030700 & 1.299911 & -0.691803 \\ \text { C } & 0 & 1.319702 & -0.889526 & -1.288330 \\ \text { H } & 0 & -2.709351 & 1.356476 & 1.255081 \\ \text { H } & 0 & -2.205902 & 1.097031 & -1.150406 \\ \text { H } & 0 & -1.712662 & -0.672775 & -0.863861 \\ \text { H } & 0 & -0.094055 & 1.664459 & 0.689758 \\ \text { H } & 0 & -0.482651 & -1.159973 & 3.183975 \\ \text { H } & 0 & -0.749832 & 0.871338 & 2.922791 \\ \text { H } & 0 & 0.347031 & 0.999802 & -1.753418 \\ \text { H } & 0 & 1.977829 & -0.854675 & -2.161057 \\ \text { H } & 0 & 1.854080 & -1.344620 & -0.451706 \\ \text { H } & 0 & 0.455475 & -1.516617 & -1.527893\end{array}$

$\mathrm{HF}=-471.6644173$

Zero-point correction=

.151169 (Hartree/Particle)

$1 \mathrm{~A}^{\prime}$

C 0

2.548676

$-0.051559$

0.987564

C 0

2.195923

$-0.171036$

$-0.333069$

C 0

0.104050

0.235306

0.442520

O 0

$-0.037277$

$-0.659851$

1.338806

B 0

0.908005

$-0.608932$

2.572067

$\begin{array}{lll}\mathrm{O} & 0 & 2.227051\end{array}$

$-0.954025$

1.872787

C $0 \quad-0.645981$

0.065872

$-0.862808$

F 0

$-0.472595$

$-1.225327$

$-1.343460$

C 0

$-2.138962$

0.311386

$-0.651840$

$\mathrm{H} O$

2.951111

0.899979

1.360703

H $0 \quad 2.486450$

0.601028

$-1.040756$

$\begin{array}{ll}\mathrm{H} & 0\end{array}$

1.869095

$-1.126465$

0.303787

1.268280

$-0.727539$

0.749893 
Journal of the American Chemical Society

$\begin{array}{rrrrr}\mathrm{H} & \mathrm{O} & 0.603958 & -1.483780 & 3.327835 \\ \mathrm{H} & 0 & 0.917908 & 0.529831 & 2.993683 \\ \mathrm{H} & 0 & -0.238647 & 0.764069 & -1.603424 \\ \mathrm{H} & 0 & -2.664932 & 0.172562 & -1.600952 \\ \mathrm{H} & 0 & -2.325272 & 1.330170 & -0.295410 \\ \mathrm{H} & 0 & -2.528763 & -0.398453 & 0.082657\end{array}$

$\mathrm{HF}=-471.6659095$

Zero-point correction $=\quad .151021$ (Hartree/Particle)

\begin{tabular}{llrrr}
\multicolumn{1}{l}{$\mathbf{1 B}^{\prime}$} & & & \\
$\mathrm{C}$ & 0 & 1.930557 & 0.230362 & 1.038849 \\
$\mathrm{C}$ & 0 & 1.562897 & 0.120087 & -0.279907 \\
$\mathrm{C}$ & 0 & -0.494064 & 0.467697 & 0.546295 \\
$\mathrm{O}$ & 0 & -0.619705 & -0.437439 & 1.442856 \\
$\mathrm{~B}$ & 0 & 0.356186 & -0.401062 & 2.650955 \\
$\mathrm{O}$ & 0 & 1.665878 & -0.706955 & 1.904480 \\
$\mathrm{C}$ & 0 & -1.336014 & 0.335617 & -0.710052 \\
$\mathrm{~F}$ & 0 & -0.804367 & 1.184875 & -1.679718 \\
$\mathrm{C}$ & 0 & -1.506790 & -1.066605 & -1.258591 \\
$\mathrm{H}$ & 0 & 2.267532 & 1.199249 & 1.431534 \\
$\mathrm{H}$ & 0 & 1.781433 & 0.916428 & -0.983170 \\
$\mathrm{H}$ & 0 & 1.326706 & -0.859665 & -0.679825 \\
$\mathrm{H}$ & 0 & -0.282500 & 1.496307 & 0.854874 \\
$\mathrm{H}$ & 0 & 0.089249 & -1.302307 & 3.389847 \\
$\mathrm{H}$ & 0 & 0.365219 & 0.723816 & 3.105164 \\
$\mathrm{H}$ & 0 & -2.320847 & 0.748383 & -0.436600 \\
$\mathrm{H}$ & 0 & -2.213303 & -1.051407 & -2.093277 \\
$\mathrm{H}$ & 0 & -1.890198 & -1.727997 & -0.477249 \\
$\mathrm{H}$ & 0 & -0.554932 & -1.465927 & -1.618271
\end{tabular}

$\mathrm{HF}=-471.6668368$

Zero-point correction=

.151033 (Hartree/Particle)

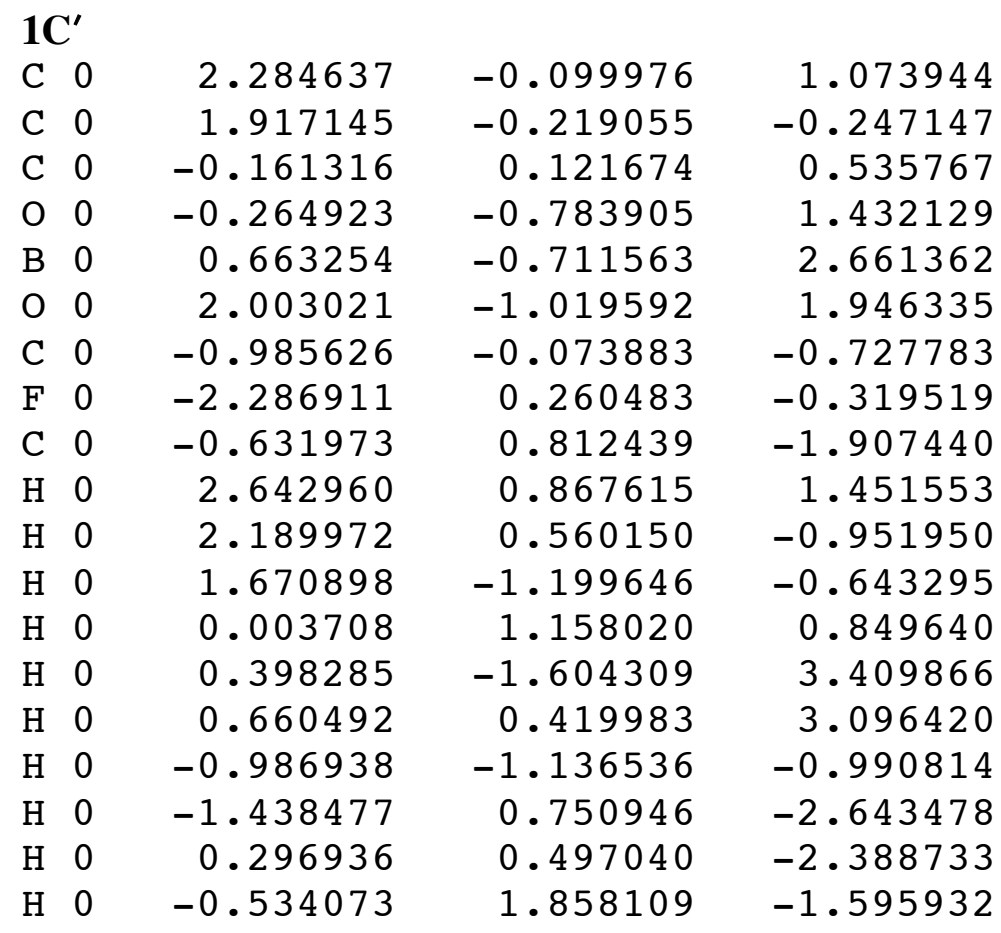


Zero-point correction $=\quad .151003$ (Hartree/Particle)

Coordinates for transition state geometries for the reaction of acetaldehyde enolborane (7) with 2chloropropanal (2)

\begin{tabular}{|c|c|c|c|}
\hline \multicolumn{4}{|l|}{4} \\
\hline \\
\hline \\
\hline \multirow{2}{*}{$\begin{array}{l}0 \\
0\end{array}$} & \\
\hline & 0.328903 & -0.366806 & 1.546082 \\
\hline & -0.660706 & -0.280197 & 2.727264 \\
\hline \multirow{2}{*}{$\begin{array}{ll}0 & 0 \\
C & 0\end{array}$} & -1.968781 & -0.600815 & 1.970367 \\
\hline & 1.166748 & 0.3 & 79681 \\
\hline $\begin{array}{lll}\mathrm{C} & 0 \\
\mathrm{Cl} & 0\end{array}$ & 1.232742 & -1.309021 & -1.21415 \\
\hline C 0 & 0.891129 & 1.381104 & -1.702255 \\
\hline $\begin{array}{ll}\mathrm{H} & 0\end{array}$ & -2.606689 & 1.268417 & 1.412796 \\
\hline H 0 & -2.023483 & 0.938126 & -0.954544 \\
\hline \multirow{2}{*}{$\begin{array}{ll}\mathrm{H} & 0 \\
\mathrm{H} & 0\end{array}$} & -1.4927 & -0.80 & -0.604263 \\
\hline & 0.059555 & 1.557114 & 0.920105 \\
\hline $\begin{array}{ll}\mathrm{H} & 0 \\
\mathrm{H} & \end{array}$ & -0.421753 & -1.155640 & 3.504440 \\
\hline H 0 & -0.673416 & 0.861130 & \\
\hline \multirow{2}{*}{$\begin{array}{ll}\mathrm{H} & 0\end{array}$} & 2.171738 & 0.566589 & -0.175552 \\
\hline & 1.703690 & 1.354233 & -2.432327 \\
\hline $\begin{array}{ll}\mathrm{H} & 0 \\
\mathrm{H} & 0\end{array}$ & -0.042725 & 1.152740 & -2.220657 \\
\hline $\begin{array}{lll}\mathrm{H} & 0 \\
\mathrm{H} & 0\end{array}$ & 0.830124 & 2.398102 & -1.295578 \\
\hline
\end{tabular}

.149426 (Hartree/Particle)

2B

$\begin{array}{llrrr}\text { C } & 0 & -2.272797 & -0.024200 & 0.670898 \\ \text { C } & 0 & -1.815918 & -0.174271 & -0.617859 \\ \text { C } & 0 & 0.193481 & 0.127060 & 0.297821 \\ \text { O } & 0 & 0.225067 & -0.763794 & 1.219025 \\ \text { B } & 0 & -0.801819 & -0.660370 & 2.374725 \\ \text { O } & 0 & -2.086945 & -0.943634 & 1.572617 \\ \text { C } & 0 & 1.106140 & -0.132416 & -0.888260 \\ \text { Cl } & 0 & 0.836090 & 1.131729 & -2.169708 \\ \text { C } & 0 & 2.569016 & -0.111725 & -0.436920 \\ \text { H } & 0 & -2.616486 & 0.960770 & 1.014648 \\ \text { H } & 0 & -1.989639 & 0.602753 & -1.354416 \\ \text { H } & 0 & -1.571808 & -1.168549 & -0.978271 \\ \text { H } & 0 & 0.033340 & 1.174423 & 0.571472 \\ \text { H } & 0 & -0.604492 & -1.549652 & 3.148727 \\ \text { H } & 0 & -0.795898 & 0.476257 & 2.797821 \\ \text { H } & 0 & 0.864059 & -1.092758 & -1.345230 \\ \text { H } & 0 & 3.226532 & -0.291931 & -1.291412 \\ \text { H } & 0 & 2.830444 & 0.853012 & 0.008362 \\ \text { H } & 0 & 2.721649 & -0.898560 & 0.308136\end{array}$

$\mathrm{HF}=-832.0309198$

Zero-point correction $=\quad .149588$ (Hartree/Particle)

2C

$\begin{array}{lllll}\text { C } 0 & -2.436035 & 0.371490 & 0.534821\end{array}$ 
Journal of the American Chemical Society

Supporting Information Page 18

$\begin{array}{llccr}\text { C } & 0 & -1.956711 & 0.221420 & -0.754425 \\ \text { C } & 0 & 0.002151 & 0.537750 & 0.084106 \\ \text { O } & 0 & 0.081573 & -0.345261 & 1.017929 \\ \text { B } & 0 & -0.882782 & -0.239578 & 2.198044 \\ \text { O } & 0 & -2.228104 & -0.528870 & 1.437836 \\ \text { C } 0 & 0.852814 & 0.324219 & -1.156113 \\ \text { Cl } 0 & 0 & 2.410858 & 1.259674 & -0.847366 \\ \text { C } 0 & 1.177948 & -1.127182 & -1.467865 \\ \text { H } & 0 & -2.805100 & 1.353531 & 0.863831 \\ \text { H } & 0 & -2.158279 & 0.990677 & -1.495316 \\ \text { H } & 0 & -1.743866 & -0.779160 & -1.115860 \\ \text { H } & 0 & -0.152954 & 1.581772 & 0.369461 \\ \text { H } & 0 & -0.679504 & -1.131470 & 2.966354 \\ \text { H } & 0 & -0.889923 & 0.894394 & 2.623077 \\ \text { H } & 0 & 0.402740 & 0.836731 & -2.006897 \\ \text { H } & 0 & 1.837143 & -1.185928 & -2.337112 \\ \text { H } & 0 & 1.661850 & -1.605728 & -0.614456 \\ \text { H } & 0 & 0.257706 & -1.677475 & -1.692795\end{array}$

$\mathrm{HF}=-832.0305140$

Zero-point correction=

.149727 (Hartree/Particle)

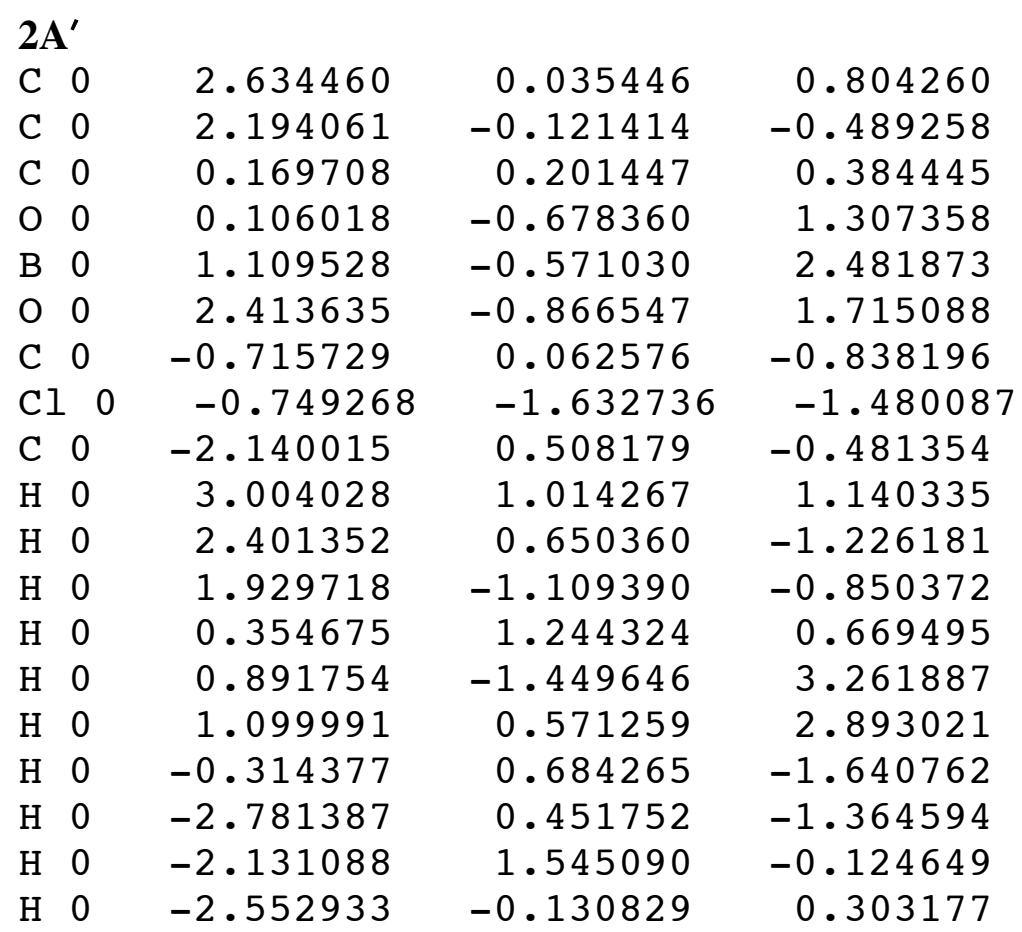

$\mathrm{HF}=-832.0282438$

Zero-point correction $=\quad .149589$ (Hartree/Particle)

2B'

C 0

1.828354

0.275452

0.628235

C $0 \quad 1.363190$

0.112274

$-0.656082$

$\begin{array}{lll}\text { C } & 0 & -0.635757\end{array}$

$\begin{array}{lll}\mathrm{O} & 0 & -0.668579\end{array}$

0.434464

0.279175

B $0 \quad 0.373932$

$-0.448196$

1.209305

O 001.650314

C $0 \quad-1.609024$

$-0.346298$

2.352112

$-0.636963$

1.538223

0.216721

$-0.868820$

Cl $0 \quad-1.315826$

1.443222

$-2.182007$ 
Journal of the American Chemical Society

Supporting Information Page 19

$\begin{array}{rrrrr}\text { C } & 0 & -1.683640 & -1.197144 & -1.423600 \\ \text { H } & 0 & 2.173843 & 1.263306 & 0.961929 \\ \text { H } & 0 & 1.529100 & 0.882996 & -1.401123 \\ \text { H } & 0 & 1.130081 & -0.888260 & -1.003403 \\ \text { H } & 0 & -0.445313 & 1.478043 & 0.545074 \\ \text { H } & 0 & 0.181580 & -1.232849 & 3.130524 \\ \text { H } & 0 & 0.377823 & 0.791275 & 2.772430 \\ \text { H } & 0 & -2.584778 & 0.498047 & -0.453384 \\ \text { H } & 0 & -2.535370 & -1.290894 & -2.101883 \\ \text { H } & 0 & -1.809372 & -1.897797 & -0.592468 \\ \text { H } & 0 & -0.775803 & -1.459641 & -1.972031\end{array}$

$\mathrm{HF}=-832.0300403$

Zero-point correction=

.149630 (Hartree/Particle)

$\begin{array}{lllll}\mathbf{2 C}^{\prime} & & & \\ \mathrm{C} & 0 & 2.426270 & 0.102203 & 0.630142 \\ \mathrm{C} & 0 & 1.942551 & -0.061081 & -0.654938 \\ \mathrm{C} & 0 & -0.044708 & 0.233688 & 0.202866 \\ \mathrm{O} & 0 & -0.073593 & -0.649445 & 1.137115 \\ \mathrm{~B} & 0 & 0.892900 & -0.509109 & 2.308807 \\ \mathrm{O} & 0 & 2.235657 & -0.791412 & 1.543700 \\ \mathrm{C} & 0 & -0.932159 & -0.024506 & -1.000214 \\ \mathrm{Cl} & 0 & -2.632828 & 0.375092 & -0.415268 \\ \mathrm{C} & 0 & -0.645355 & 0.802444 & -2.244965 \\ \mathrm{H} & 0 & 2.793289 & 1.089127 & 0.946365 \\ \mathrm{H} & 0 & 2.151718 & 0.700912 & -1.399323 \\ \mathrm{H} & 0 & 1.720535 & -1.063705 & -1.007690 \\ \mathrm{H} & 0 & 0.088943 & 1.284576 & 0.478897 \\ \mathrm{H} & 0 & 0.708542 & -1.390579 & 3.093491 \\ \mathrm{H} & 0 & 0.885315 & 0.632187 & 2.714996 \\ \mathrm{H} & 0 & -0.950684 & -1.095703 & -1.206177 \\ \mathrm{H} & 0 & -1.456451 & 0.679184 & -2.966095 \\ \mathrm{H} & 0 & 0.283646 & 0.474564 & -2.719894 \\ \text { H } & 0 & -0.563553 & 1.866821 & -2.002167\end{array}$

$\mathrm{HF}=-832.0281132$

Zero-point correction $=\quad .149672$ (Hartree/Particle)

Coordinates for transition state geometries for the reaction of acetaldehyde enolborane (7) with 2methoxypropanal (3)

\begin{tabular}{|c|c|c|c|}
\hline $\begin{array}{l}3 B_{1} \\
\text { C }\end{array}$ & 2.301361 & 1.274887 & -0.086212 \\
\hline $\mathrm{C}$ & 1.210358 & 1.600952 & 0.684448 \\
\hline $\mathrm{C}$ & 0.326263 & -0.238449 & -0.160706 \\
\hline 0 & 1.197708 & -1.141235 & 0.117828 \\
\hline B & 2.599045 & -1.010498 & -0.51071 \\
\hline $\mathrm{O}$ & 3.074646 & 0.270844 & 0.210480 \\
\hline C & -0.979614 & -0.282043 & 0.624176 \\
\hline $\mathrm{O}$ & -1.743164 & 0.904160 & 0.45744 \\
\hline & -1.731674 & -1.579819 & 0.28289 \\
\hline C & -2.461761 & 1.052063 & -0.75845 \\
\hline $\mathrm{H}$ & 2.415817 & 1.724091 & -1.08261 \\
\hline & 0.573349 & 2.437027 & 0.41699 \\
\hline
\end{tabular}


Journal of the American Chemical Society

$\begin{array}{rrrrr}\mathrm{H} & 0 & 1.151947 & 1.231049 & 1.702744 \\ \mathrm{H} & 0 & 0.279129 & 0.129089 & -1.191254 \\ \mathrm{H} & 0 & 3.273727 & -1.936539 & -0.166473 \\ \mathrm{H} & 0 & 2.477310 & -0.811569 & -1.702759 \\ \mathrm{H} & 0 & -0.729904 & -0.307236 & 1.688904 \\ \mathrm{H} & 0 & -2.685837 & -1.599686 & 0.819473 \\ \mathrm{H} & 0 & -1.929611 & -1.680206 & -0.789322 \\ \mathrm{H} & 0 & -1.129974 & -2.437027 & 0.595810 \\ \mathrm{H} & 0 & -2.897720 & 2.053497 & -0.727631 \\ \mathrm{H} & 0 & -1.819458 & 0.980331 & -1.647919 \\ \mathrm{H} & 0 & -3.273743 & 0.319260 & -0.853439\end{array}$

$\mathrm{HF}=-486.9488691$

Zero-point correction=

.191718 (Hartree/Particle)

\begin{tabular}{|c|c|c|c|}
\hline $3 \mathbf{B}_{2}$ & & & \\
\hline C 0 & 2.419861 & 1.352036 & $\begin{array}{r}-0.21588 \\
0.287075\end{array}$ \\
\hline $\mathrm{C}$ & 1.201797 & 1.742813 & 0.287079 \\
\hline 0 & 0.556488 & -0.296677 & -0.27760 \\
\hline 0 & 1.408966 & -1.053436 & 0.31243 \\
\hline 0 & 2.890915 & -0.937805 & -0.101944 \\
\hline 0 & 3.175446 & 0.493027 & 0.40577 \\
\hline 0 & -0.856842 & -0.290817 & 0.27465 \\
\hline $\mathrm{O}$ & -1.551392 & 0.749634 & $-0.39280^{\circ}$ \\
\hline $\mathrm{C}$ & -1.481323 & -1.674988 & 0.02539 \\
\hline $\mathrm{C}$ & -2.785400 & 1.112656 & 0.19970 \\
\hline $\mathrm{H}$ & 2.679932 & 1.595078 & -1.25528 \\
\hline $\mathrm{H}$ & 0.582565 & 2.448914 & -0.25483 \\
\hline $\mathrm{H}$ & 0.987579 & 1.582779 & 1.33888 \\
\hline 0 & 0.641937 & -0.139328 & -1.35794 \\
\hline 0 & 3.536896 & -1.722290 & 0.52955 \\
\hline $\mathrm{H}$ & 2.965225 & -0.987198 & -1.31260 \\
\hline 0 & -0.816513 & -0.101334 & 1.35794 \\
\hline $\mathrm{H}$ & -2.498856 & -1.715714 & 0.42636 \\
\hline $\mathrm{H}$ & -1.523956 & -1.886612 & -1.04832 \\
\hline 0 & -0.885406 & -2.448929 & 0.51681 \\
\hline $\mathrm{H}$ & -3.149658 & 1.984314 & -0.34921 \\
\hline $\mathrm{H}$ & -3.536911 & 0.314148 & 0.12924 \\
\hline U & -2.658920 & 1.383804 & 1.25933 \\
\hline
\end{tabular}

$\mathrm{HF}=-486.9528734$

Zero-point correction $=\quad .191433$ (Hartree/Particle)

$\begin{array}{rrrrr}\mathbf{3 C}_{1} & & & \\ \mathrm{C} & 0 & -2.785019 & 0.543182 & 0.702591 \\ \mathrm{C} & 0 & -1.886612 & 1.571487 & 0.509338 \\ \mathrm{C} & 0 & -0.356476 & 0.031647 & 0.627548 \\ \mathrm{O} & 0 & -0.716309 & -0.819839 & -0.271423 \\ \mathrm{~B} & 0 & -2.053223 & -1.550369 & -0.082062 \\ \mathrm{O} & 0 & -3.017288 & -0.335938 & -0.222580 \\ \mathrm{C} & 0 & 0.921158 & 0.838608 & 0.390167 \\ \text { O } & 0 & 1.976074 & 0.115097 & 1.028687 \\ \text { C } & 0 & 1.189590 & 1.178726 & -1.073837 \\ \mathrm{C} & 0 & 2.443054 & -1.061295 & 0.372589 \\ \mathrm{H} & 0 & -3.154480 & 0.328705 & 1.715225\end{array}$


Journal of the American Chemical Society

$\begin{array}{rrrrr}\mathrm{H} & 0 & -1.719604 & 2.298691 & 1.299377 \\ \mathrm{H} & 0 & -1.631546 & 1.861328 & -0.504318 \\ \mathrm{H} & 0 & -0.524963 & -0.231491 & 1.676956 \\ \mathrm{H} & 0 & -2.231873 & -2.298691 & -0.997894 \\ \mathrm{H} & 0 & -2.109528 & -2.003723 & 1.041321 \\ \mathrm{H} & 0 & 0.854523 & 1.758621 & 0.979172 \\ \mathrm{H} & 0 & 2.183609 & 1.627335 & -1.165192 \\ \mathrm{H} & 0 & 1.131332 & 0.296539 & -1.715225 \\ \mathrm{H} & 0 & 0.455750 & 1.903488 & -1.439362 \\ \mathrm{H} & 0 & 3.154480 & -1.520355 & 1.063034 \\ \mathrm{H} & 0 & 1.634323 & -1.772018 & 0.163239 \\ \mathrm{H} & 0 & 2.961609 & -0.826920 & -0.565933\end{array}$

$\mathrm{HF}=-486.9502145$

Zero-point correction=

$3 \mathrm{C}_{2}$

C 0

C 0

C 0

$\begin{array}{lll}0 & 0\end{array}$

B 0

O 0

C 0

O 0

C 0

C 0

$\mathrm{H} \quad \mathrm{O}$

$\mathrm{H} \quad \mathrm{O}$

$\mathrm{H} \quad \mathrm{O}$

$\begin{array}{lll}\mathrm{H} & \mathrm{O}\end{array}$

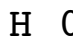

$\mathrm{H} \quad \mathrm{O}$

$\mathrm{H} \quad \mathrm{O}$

$\mathrm{H} \quad \mathrm{O}$

$\mathrm{H} \quad \mathrm{O}$

$\mathrm{H} O$

$\mathrm{H} \quad \mathrm{O}$

$\mathrm{H} \quad 0$

$\mathrm{H} \quad 0$
2.665573

1.708679

0.516739

1.292145

2. 583344

3.333908

$-0.708725$

$-1.749039$

$-0.544693$

$-3.048828$

2.716476

1.172165

1.755341

0.356308

3.180603

2.345917

$-0.927567$

$-1.470900$

$-0.304352$

0.257202

$-3.729095$

$-3.359970$

$-3.119537$
$-0.510086$

$-1.346420$

0.429962

1.204742

1.689484

0.317413

$-0.152634$

0.755951

$-0.263550$

0.193222

$-0.380325$

$-2.036850$

$-1.582489$

0.654037

2.396194

2.114487

$-1.137741$

$-0.629776$

0.714508

$-0.960297$

0.974700

$-0.124252$

$-0.671143$
.191921 (Hartree/Particle)
1.626633
1.086487
0.801712
0.127823
0.784164
0.888947
0.096268
0.455856
$-1.417236$
0.447235
2.717255
1.731949
0.028534
1.860718
0.026900
1.894135
0.535614
$-1.870758$
$-1.839218$
$-1.681763$
0.794159
$-0.558304$
1.126236

$\mathrm{HF}=-486.9491133$

Zero-point correction

.191367 (Hartree/Particle)

$\begin{array}{rrrrr}\mathbf{3 C}_{3} & & & \\ \mathrm{C} & 0 & -2.692734 & 0.892990 & -0.658554 \\ \mathrm{C} & 0 & -1.681503 & 0.504883 & -1.514709 \\ \mathrm{C} & 0 & -0.457916 & 0.294220 & 0.253448 \\ \mathrm{O} & 0 & -1.143997 & -0.503464 & 0.995682 \\ \mathrm{~B} & 0 & -2.501526 & -0.024734 & 1.505264 \\ \mathrm{O} & 0 & -3.265991 & 0.050217 & 0.140076 \\ \mathrm{C} & 0 & 0.847488 & -0.230347 & -0.350143 \\ \mathrm{O} & 0 & 1.886856 & 0.127121 & 0.562057 \\ \mathrm{C} & 0 & 0.873093 & -1.743866 & -0.509415 \\ \mathrm{C} & 0 & 2.463120 & 1.403503 & 0.343613\end{array}$


Journal of the American Chemical Society

Supporting Information Page 22

$\begin{array}{rrrrr}\mathrm{H} & 0 & -2.875946 & 1.964005 & -0.489502 \\ \mathrm{H} & 0 & -1.230331 & 1.236237 & -2.180283 \\ \mathrm{H} & 0 & -1.598923 & -0.541229 & -1.790558 \\ \mathrm{H} & 0 & -0.448975 & 1.354400 & 0.528809 \\ \mathrm{H} & 0 & -3.003860 & -0.874191 & 2.180283 \\ \mathrm{H} & 0 & -2.392273 & 1.087311 & 1.977249 \\ \mathrm{H} & 0 & 1.023804 & 0.264481 & -1.316071 \\ \mathrm{H} & 0 & 1.837906 & -2.046677 & -0.925369 \\ \mathrm{H} & 0 & 0.737244 & -2.224518 & 0.461792 \\ \mathrm{H} & 0 & 0.077072 & -2.087509 & -1.176636 \\ \mathrm{H} & 0 & 3.265991 & 1.511627 & 1.076736 \\ \mathrm{H} & 0 & 2.887054 & 1.484146 & -0.669235 \\ \mathrm{H} & 0 & 1.745773 & 2.224518 & 0.489120\end{array}$

$\mathrm{HF}=-486.9497481$

Zero-point correction $=\quad .191508$ (Hartree/Particle)

Coordinates for transition state geometries for the reaction of acetaldehyde enolborane (7) with 2thiomethylpropanal (4)

\begin{tabular}{|c|c|c|c|}
\hline $\begin{array}{l}\mathbf{4} \mathbf{B}_{1} \\
\text { C }\end{array}$ & 2.354095 & 1.429138 & -0.02244 \\
\hline C & 1.291931 & 1.666992 & 0.822403 \\
\hline $\mathrm{C}$ & 0.441513 & -0.170212 & -0.004990 \\
\hline $\mathrm{O}$ & 1.375504 & -1.034149 & 0.199295 \\
\hline B & 2.718262 & -0.834106 & -0.51582 \\
\hline 0 & 3.193619 & 0.462708 & 0.19491 \\
\hline C & -0.823105 & -0.329895 & 0.82983 \\
\hline$S$ & -2.062073 & 1.001556 & 0.51486 \\
\hline C & -1.384200 & -1.751663 & 0.64222 \\
\hline C & -2.769608 & 0.529449 & -1.10583 \\
\hline $\mathrm{H}$ & 2.383392 & 1.916214 & -1.00727 \\
\hline $\mathrm{H}$ & 0.604950 & 2.482788 & 0.62442 \\
\hline $\mathrm{H}$ & 1.331131 & 1.272018 & 1.83262 \\
\hline $\mathrm{H}$ & 0.312347 & 0.211136 & -1.02330 \\
\hline $\mathrm{H}$ & 3.457092 & -1.731018 & -0.23210 \\
\hline $\mathrm{H}$ & 2.514435 & -0.619415 & -1.69323 \\
\hline $\mathrm{H}$ & -0.569962 & -0.203613 & 1.88665 \\
\hline $\mathrm{H}$ & -2.287628 & -1.877000 & 1.24612 \\
\hline $\mathrm{H}$ & -1.632996 & -1.957336 & -0.40376 \\
\hline $\mathrm{H}$ & -0.634827 & -2.482788 & 0.95803 \\
\hline $\mathrm{H}$ & -3.457092 & 1.336960 & -1.37115 \\
\hline $\mathrm{H}$ & -2.007492 & 0.456146 & -1.88665 \\
\hline & -3.334061 & -0.404205 & -1.04847 \\
\hline
\end{tabular}

$\mathrm{HF}=-809.9318335$

Zero-point correction=

.187909 (Hartree/Particle)
$4 B_{2}$
C 0
2.667969
1.482300
$-0.200928$
C $0 \quad 1.437317$
1.770554
C $0 \quad 0.843140$
$-0.220123$
$\begin{array}{ll}\mathrm{O} & 0\end{array}$
1.740891
$-0.981689$
0.349380
$-0.345900$
0.177521
B $0 \quad 3.198257$
$-0.801300$
$-0.264236$
O 0
3. 465668
0.608597
0.332947 
Journal of the American Chemical Society

Supporting Information Page 23

$\begin{array}{rrrrr}\mathrm{C} & 0 & -0.559097 & -0.364487 & 0.223923 \\ \mathrm{~S} & 0 & -1.652222 & 0.928177 & -0.517900 \\ \mathrm{C} & 0 & -1.043564 & -1.797806 & -0.067062 \\ \mathrm{C} & 0 & -3.204987 & 0.625656 & 0.394897 \\ \mathrm{H} & 0 & 2.901200 & 1.829773 & -1.217239 \\ \mathrm{H} & 0 & 0.795959 & 2.516556 & -0.107269 \\ \mathrm{H} & 0 & 1.256866 & 1.524963 & 1.391037 \\ \mathrm{H} & 0 & 0.915375 & -0.003555 & -1.417328 \\ \mathrm{H} & 0 & 3.885330 & -1.602615 & 0.298477 \\ \mathrm{H} & 0 & 3.248428 & -0.762802 & -1.476044 \\ \mathrm{H} & 0 & -0.515625 & -0.210068 & 1.306992 \\ \mathrm{H} & 0 & -2.044205 & -1.958435 & 0.344482 \\ \mathrm{H} & 0 & -1.080963 & -1.988098 & -1.144547 \\ \mathrm{H} & 0 & -0.360641 & -2.516556 & 0.395737 \\ \mathrm{H} & 0 & -3.885345 & 1.431870 & 0.108917 \\ \mathrm{H} & 0 & -3.663223 & -0.329407 & 0.126572 \\ \mathrm{H} & 0 & -3.043457 & 0.672211 & 1.476044\end{array}$

$\mathrm{HF}=-809.9318358$

Zero-point correction=

.187948 (Hartree/Particle)

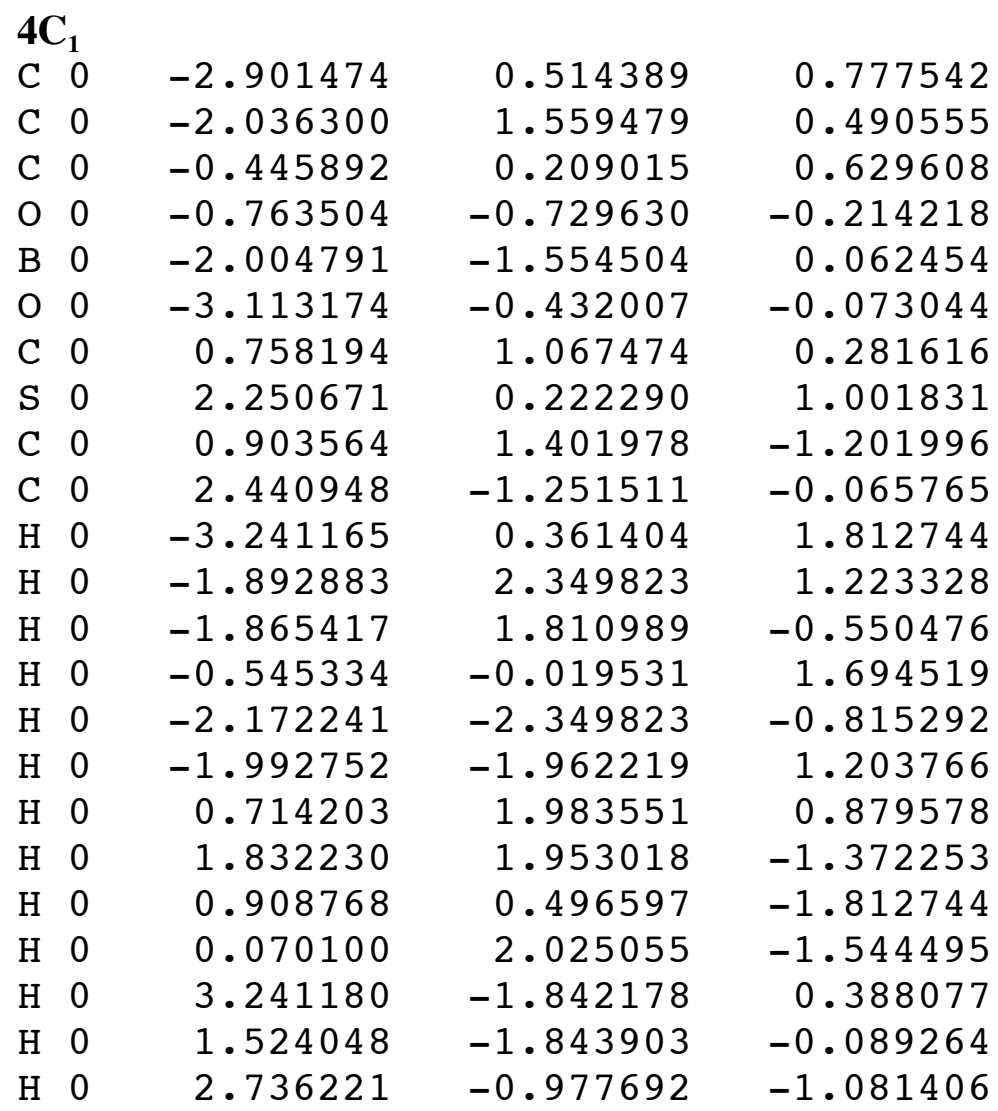

$\mathrm{HF}=-809.9370623$

Zero-point correction=

.188053 (Hartree/Particle)
$4 \mathrm{C}_{2}$
C 0
3.327652
$-0.612747$
1.004608
C 0
2. 304520
$-1.497467$
0.704575
C 0
1.026382
0.175369
0.503342
O 0
1.633500
0.921616
$-0.364182$
B 0
2.984833
1.502945
0.007339 


$\begin{array}{rrrrr}\mathrm{O} & 0 & 3.826385 & 0.165924 & 0.104416 \\ \mathrm{C} & 0 & -0.281326 & -0.460846 & 0.067810 \\ \mathrm{~S} & 0 & -1.572723 & 0.848175 & 0.401000 \\ \mathrm{C} & 0 & -0.311539 & -0.932510 & -1.384949 \\ \mathrm{C} & 0 & -2.984970 & -0.217438 & 0.861633 \\ \mathrm{H} & 0 & 3.570953 & -0.403946 & 2.057022 \\ \mathrm{H} & 0 & 1.916168 & -2.149902 & 1.482727 \\ \mathrm{H} & 0 & 2.194977 & -1.827072 & -0.322662 \\ \mathrm{H} & 0 & 1.055161 & 0.491104 & 1.550186 \\ \mathrm{H} & 0 & 3.417007 & 2.149887 & -0.900314 \\ \mathrm{H} & 0 & 2.932266 & 2.019058 & 1.102386 \\ \mathrm{H} & 0 & -0.509552 & -1.278763 & 0.758759 \\ \mathrm{H} & 0 & -1.311905 & -1.289780 & -1.646606 \\ \mathrm{H} & 0 & -0.046906 & -0.113663 & -2.057022 \\ \mathrm{H} & 0 & 0.391434 & -1.757233 & -1.550049 \\ \mathrm{H} & 0 & -3.826400 & 0.452972 & 1.056366 \\ \mathrm{H} & 0 & -3.260483 & -0.893677 & 0.047211 \\ \mathrm{H} & 0 & -2.776917 & -0.791870 & 1.769287\end{array}$

$\mathrm{HF}=-809.9328277$

Zero-point correction=

.187854 (Hartree/Particle)

\begin{tabular}{|c|c|c|c|}
\hline \multicolumn{4}{|l|}{$4 C_{3}$} \\
\hline C 0 & -2.822220 & 1.000275 & -0.713531 \\
\hline $\mathrm{C}$ & -1.599747 & 1.454956 & -1.185150 \\
\hline $\mathrm{C}$ & -0.657959 & 0.038208 & 0.046143 \\
\hline $\mathrm{O}$ & -1.207794 & -1.081329 & -0.306671 \\
\hline B & -2.683884 & -1.268967 & -0.043808 \\
\hline 0 & -3.273270 & -0.166000 & -1.02587 \\
\hline C & 0.806137 & 0.237366 & -0.31079 \\
\hline S & 1.822418 & -0.403168 & 1.10931 \\
\hline C & 1.231064 & -0.494995 & -1.58415 \\
\hline C & 1.754044 & 1.018768 & 2.25648 \\
\hline $\mathrm{H}$ & -3.316116 & 1.539490 & 0.10913 \\
\hline $\mathrm{H}$ & -1.261703 & 2.451385 & -0.91087 \\
\hline $\mathrm{H}$ & -1.217392 & 1.040588 & -2.11187 \\
\hline $\mathrm{H}$ & -0.967682 & 0.465042 & 1.00541 \\
\hline $\mathrm{H}$ & -3.043137 & -2.339554 & -0.43620 \\
\hline $\mathrm{H}$ & -2.953003 & -0.959076 & 1.09680 \\
\hline $\mathrm{H}$ & 1.021454 & 1.307602 & -0.38772 \\
\hline $\mathrm{H}$ & 2.284576 & -0.297226 & -1.79734 \\
\hline $\mathrm{H}$ & 1.085831 & -1.573242 & -1.47998 \\
\hline $\mathrm{H}$ & 0.634491 & -0.163910 & -2.44044 \\
\hline $\mathrm{H}$ & 2.378891 & 0.752502 & 3.11315 \\
\hline $\mathrm{H}$ & 2.161041 & 1.922897 & 1.79315 \\
\hline $\mathrm{H}$ & 0.739761 & 1.209869 & 2.61862 \\
\hline
\end{tabular}

$\mathrm{HF}=-809.9338614$

Zero-point correction $=\quad .187811$ (Hartree/Particle)

Coordinates for transition state geometries for the reaction of acetaldehyde enolborane (7) with 2dimethylaminopropanal (5)

$5 \mathbf{B}_{3}$

C 0

2.307587

1.456375

$-0.092239$ 
Journal of the American Chemical Society

Supporting Information Page 25

$\begin{array}{rrrrr}\mathrm{C} & 0 & 1.117355 & 1.757355 & 0.529175 \\ \mathrm{C} & 0 & 0.491135 & -0.244675 & -0.090881 \\ \mathrm{O} & 0 & 1.415222 & -1.005188 & 0.385803 \\ \mathrm{~B} & 0 & 2.844513 & -0.823471 & -0.142319 \\ \mathrm{O} & 0 & 3.142883 & 0.595306 & 0.409744 \\ \mathrm{C} & 0 & -0.878098 & -0.331741 & 0.573486 \\ \mathrm{~N} & 0 & -1.782806 & 0.705826 & 0.061874 \\ \mathrm{C} & 0 & -1.385132 & -1.790451 & 0.450195 \\ \mathrm{C} & 0 & -2.824448 & 1.074890 & 1.012527 \\ \mathrm{C} & 0 & -2.345749 & 0.448044 & -1.260773 \\ \mathrm{H} & 0 & 2.471802 & 1.774567 & -1.131439 \\ \mathrm{H} & 0 & 0.425140 & 2.465988 & 0.088730 \\ \mathrm{H} & 0 & 1.004425 & 1.531754 & 1.584488 \\ \mathrm{H} & 0 & 0.510208 & -0.035309 & -1.165497 \\ \mathrm{H} & 0 & 3.568604 & -1.613922 & 0.389832 \\ \mathrm{H} & 0 & 2.821503 & -0.808594 & -1.356827 \\ \mathrm{H} & 0 & -0.729416 & -0.130646 & 1.639038 \\ \mathrm{H} & 0 & -2.376251 & -1.884262 & 0.906845 \\ \mathrm{H} & 0 & -1.457291 & -2.112335 & -0.593918 \\ \mathrm{H} & 0 & -0.697357 & -2.465988 & 0.964783 \\ \mathrm{H} & 0 & -3.365448 & 1.947800 & 0.630737 \\ \mathrm{H} & 0 & -3.568619 & 0.277115 & 1.197403 \\ \mathrm{H} & 0 & -2.374344 & 1.351456 & 1.971664 \\ \mathrm{H} & 0 & -2.820007 & 1.364655 & -1.628326 \\ \mathrm{H} & 0 & -1.559418 & 0.176941 & -1.971664 \\ \mathrm{H} & 0 & -3.107254 & -0.352509 & -1.273500 \\ \mathrm{H} & -1.50 & 0.3937819 & & \end{array}$

$\mathrm{HF}=-506.3937819$

Zero-point correction=

.232501 (Hartree/Particle)

$\begin{array}{rrrrr}\mathbf{5 C}_{\mathbf{1}} & & & \\ \mathrm{C} & 0 & 3.008621 & -0.901367 & -0.475677 \\ \mathrm{C} & 0 & 2.001862 & -0.880417 & -1.424545 \\ \mathrm{C} & 0 & 0.706696 & -0.158356 & 0.082474 \\ \mathrm{O} & 0 & 1.318039 & 0.892624 & 0.524475 \\ \mathrm{~B} & 0 & 2.653397 & 0.710251 & 1.218033 \\ \mathrm{O} & 0 & 3.513657 & 0.188629 & -0.000748 \\ \mathrm{C} & 0 & -0.603531 & 0.055725 & -0.696030 \\ \mathrm{~N} & 0 & -1.667297 & -0.211365 & 0.295059 \\ \mathrm{C} & 0 & -0.639938 & 1.451859 & -1.320480 \\ \mathrm{C} & 0 & -2.001892 & -1.628418 & 0.358643 \\ \mathrm{C} & 0 & -2.872681 & 0.604965 & 0.213287 \\ \mathrm{H} & 0 & 3.236389 & -1.842560 & 0.046783 \\ \mathrm{H} & 0 & 1.611100 & -1.818817 & -1.809906 \\ \mathrm{H} & 0 & 1.896973 & -0.003464 & -2.054962 \\ \mathrm{H} & 0 & 0.725922 & -1.045700 & 0.721695 \\ \mathrm{H} & 0 & 3.089737 & 1.771805 & 1.555618 \\ \mathrm{H} & 0 & 2.576508 & -0.164063 & 2.054962 \\ \mathrm{H} & 0 & -0.661224 & -0.684708 & -1.512482 \\ \mathrm{H} & 0 & -1.550705 & 1.593018 & -1.908432 \\ \mathrm{H} & 0 & -0.572296 & 2.233154 & -0.560425 \\ \mathrm{H} & 0 & 0.214706 & 1.576935 & -1.991394 \\ \mathrm{H} & 0 & -2.668518 & -1.812347 & 1.207489 \\ \mathrm{H} & 0 & -2.502792 & -1.993179 & -0.559860\end{array}$


Journal of the American Chemical Society

$\begin{array}{rrrrr}\mathrm{H} & 0 & -1.102249 & -2.233170 & 0.510330 \\ \mathrm{H} & 0 & -3.513657 & 0.356613 & 1.065704 \\ \mathrm{H} & 0 & -2.629562 & 1.666443 & 0.283554 \\ \mathrm{H} & 0 & -3.461288 & 0.439041 & -0.709335\end{array}$

$\mathrm{HF}=-506.3895042$

Zero-point correction $=\quad .23265$ (Hartree/Particle)

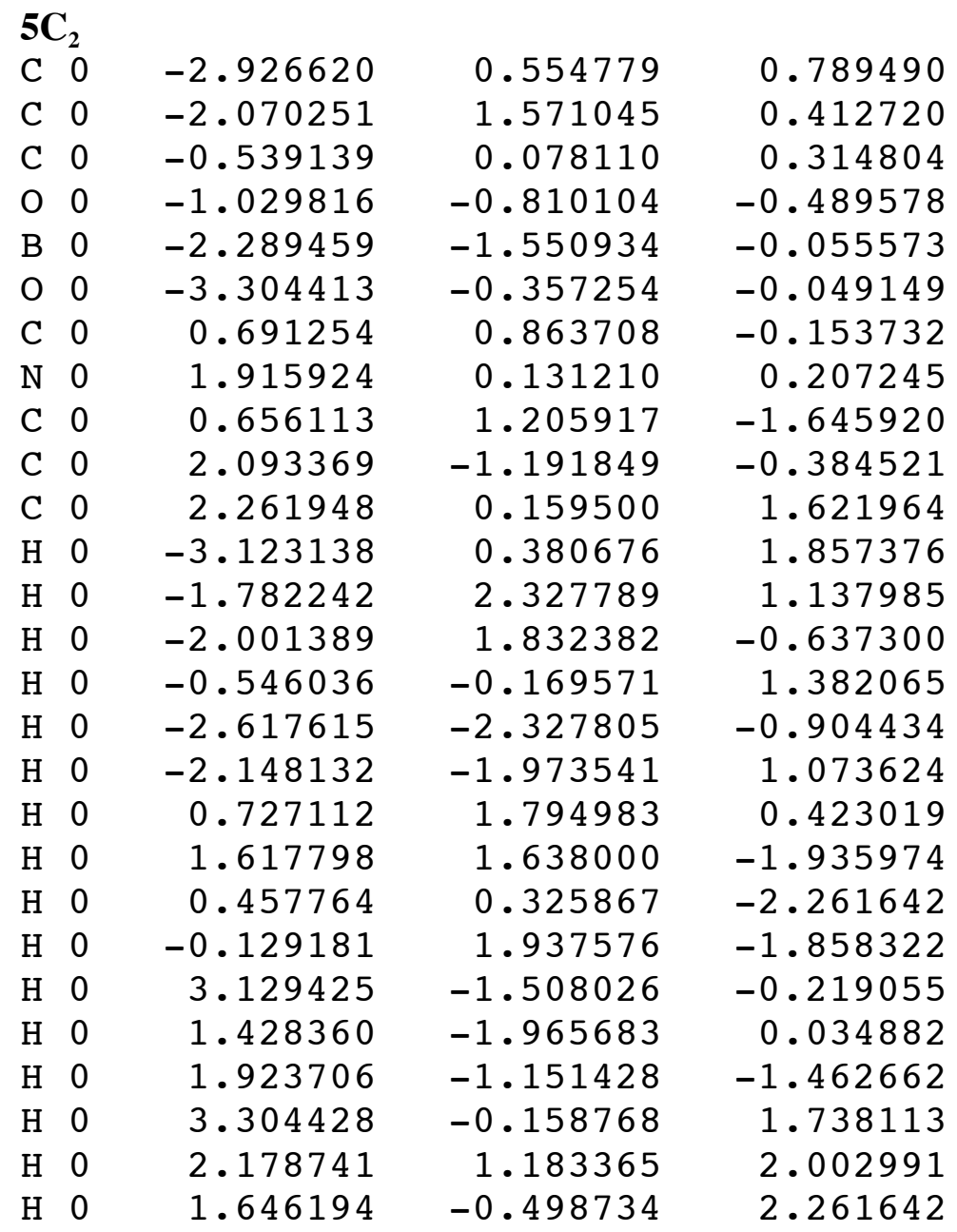

$\mathrm{HF}=-506.3951197$

Zero-point correction $=\quad .232485$ (Hartree/Particle)

$\begin{array}{rrrrr}\mathbf{5 C}_{3} & & & \\ \mathrm{C} & 0 & -3.103561 & 0.715118 & 0.821274 \\ \mathrm{C} & 0 & -2.120636 & 1.573578 & 0.364900 \\ \mathrm{C} & 0 & -0.747253 & -0.032669 & 0.597839 \\ \mathrm{O} & 0 & -1.277313 & -0.984695 & -0.101837 \\ \mathrm{~B} & 0 & -2.623337 & -1.543976 & 0.338974 \\ \mathrm{O} & 0 & -3.520187 & -0.275604 & 0.101440 \\ \mathrm{C} & 0 & 0.567627 & 0.576050 & 0.098389 \\ \mathrm{~N} & 0 & 1.632568 & -0.168503 & 0.789368 \\ \mathrm{C} & 0 & 0.673798 & 0.636429 & -1.432709 \\ \mathrm{C} & 0 & 2.845612 & 0.608170 & 1.005600 \\ \mathrm{C} & 0 & 1.921036 & -1.496292 & 0.253403 \\ \mathrm{H} & 0 & -3.383667 & 0.729614 & 1.884659 \\ \mathrm{H} & 0 & -1.800903 & 2.403015 & 0.990509 \\ \mathrm{H} & 0 & -1.965714 & 1.665756 & -0.704544\end{array}$


Journal of the American Chemical Society

Supporting Information Page 27

$\begin{array}{rrrrr}\mathrm{H} & 0 & -0.805695 & -0.110977 & 1.687546 \\ \mathrm{H} & 0 & -2.973190 & -2.403000 & -0.416840 \\ \mathrm{H} & 0 & -2.595474 & -1.806992 & 1.522293 \\ \mathrm{H} & 0 & 0.615891 & 1.593826 & 0.500931 \\ \mathrm{H} & 0 & 1.671204 & 0.980133 & -1.726334 \\ \mathrm{H} & 0 & 0.491470 & -0.341141 & -1.884674 \\ \mathrm{H} & 0 & -0.054321 & 1.337509 & -1.852997 \\ \mathrm{H} & 0 & 3.520203 & 0.045029 & 1.659866 \\ \mathrm{H} & 0 & 3.399017 & 0.840363 & 0.075912 \\ \mathrm{H} & 0 & 2.603195 & 1.552383 & 1.505081 \\ \mathrm{H} & 0 & 2.546082 & -2.038055 & 0.971817 \\ \mathrm{H} & 0 & 0.994232 & -2.058624 & 0.117400 \\ \mathrm{H} & 0 & 2.457611 & -1.475586 & -0.712067\end{array}$

$\mathrm{HF}=-506.3949679$

Zero-point correction $=\quad .232434$ (Hartree/Particle)

Coordinates for transition state geometries for the reaction of acetaldehyde enolborane (7) with 2dimethylphosphinopropanal (6)

\begin{tabular}{|c|c|c|c|c|}
\hline & & & & \\
\hline C & 0 & 2.698563 & 1.463028 & \\
\hline $\mathrm{C}$ & 0 & 1.525452 & 1.736542 & 0.58935 \\
\hline $\mathrm{C}$ & 0 & 0.867172 & -0.231613 & -0.01478 \\
\hline $\mathrm{O}$ & 0 & 1.815323 & -0.991470 & 0.42825 \\
\hline B & 0 & 3.209152 & -0.830475 & -0.16583 \\
\hline 0 & 0 & 3.555100 & 0.600098 & 0.36302 \\
\hline $\mathrm{C}$ & 0 & -0.485200 & -0.359222 & 0.67247 \\
\hline$P$ & 0 & -1.727661 & 0.923279 & 0.01757 \\
\hline $\mathrm{C}$ & 0 & -0.922867 & -1.838623 & 0.57884 \\
\hline $\mathrm{C}$ & 0 & -3.098587 & 0.692215 & 1.26026 \\
\hline C & 0 & -2.474762 & 0.048309 & -1.45253 \\
\hline $\mathrm{H}$ & 0 & 2.821564 & 1.813461 & -1.11998 \\
\hline $\mathrm{H}$ & 0 & 0.831406 & 2.474411 & 0.20127 \\
\hline $\mathrm{H}$ & 0 & 1.467300 & 1.499771 & 1.64701 \\
\hline $\mathrm{H}$ & 0 & 0.852997 & -0.037048 & -1.09399 \\
\hline $\mathrm{H}$ & 0 & 3.956711 & -1.617508 & 0.33776 \\
\hline $\mathrm{H}$ & 0 & 3.137329 & -0.816147 & -1.37779 \\
\hline $\mathrm{H}$ & 0 & -0.350189 & -0.116730 & 1.73362 \\
\hline $\mathrm{H}$ & 0 & -1.899109 & -1.986603 & 1.05117 \\
\hline $\mathrm{H}$ & 0 & -0.993500 & -2.176071 & -0.46046 \\
\hline $\mathrm{H}$ & 0 & -0.192627 & -2.474426 & 1.08740 \\
\hline $\mathrm{H}$ & 0 & -3.956711 & 1.295578 & 0.94548 \\
\hline $\mathrm{H}$ & 0 & -3.429169 & -0.346786 & 1.36909 \\
\hline $\mathrm{H}$ & 0 & -2.775513 & 1.064682 & 2.23809 \\
\hline $\mathrm{H}$ & 0 & -3.264694 & 0.687851 & -1.86111 \\
\hline $\mathrm{H}$ & 0 & -1.722000 & -0.078812 & -2.23811 \\
\hline $\mathrm{H}$ & 0 & -2.907516 & -0.929504 & -1.21624 \\
\hline
\end{tabular}

$\mathrm{HF}=-793.0122981$

Zero-point correction $=\quad .225143$ (Hartree/Particle)

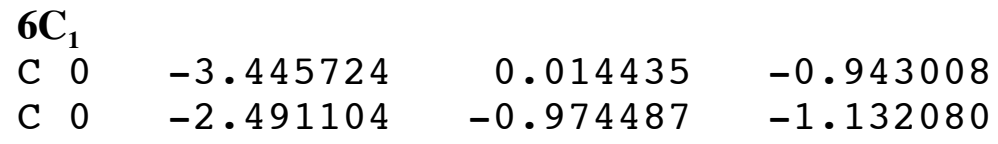


Journal of the American Chemical Society

Supporting Information Page 28

$\begin{array}{rrrrr}\text { C } & 0 & -1.107269 & 0.216873 & -0.117264 \\ \text { O } & 0 & -1.688766 & 0.446091 & 1.024689 \\ \text { B } & 0 & -2.960922 & 1.255508 & 1.023727 \\ \text { O } & 0 & -3.924011 & 0.272156 & 0.225754 \\ \text { C } & 0 & 0.147751 & -0.621400 & -0.103241 \\ \text { P } & 0 & 1.596161 & 0.488617 & 0.513260 \\ \text { C } & 0 & 0.065400 & -1.859604 & 0.798889 \\ \text { C } & 0 & 1.489243 & 1.924377 & -0.677795 \\ \text { C } & 0 & 3.001602 & -0.432861 & -0.305191 \\ \text { H } & 0 & -3.643463 & 0.736588 & -1.750000 \\ \text { H } & 0 & -2.121857 & -1.173660 & -2.135162 \\ \text { H } & 0 & -2.453186 & -1.790466 & -0.419388 \\ \text { H } & 0 & -1.103668 & 1.041336 & -0.836578 \\ \text { H } & 0 & -3.388718 & 1.363800 & 2.135162 \\ \text { H } & 0 & -2.815857 & 2.267761 & 0.370285 \\ \text { H } & 0 & 0.380173 & -0.903259 & -1.138168 \\ \text { H } & 0 & 1.039413 & -2.352615 & 0.878983 \\ \text { H } & 0 & -0.262222 & -1.580200 & 1.803192 \\ \text { H } & 0 & -0.638367 & -2.599014 & 0.401306 \\ \text { H } & 0 & 2.423294 & 2.492508 & -0.605667 \\ \text { H } & 0 & 1.355240 & 1.616745 & -1.722519 \\ \text { H } & 0 & 0.675629 & 2.599000 & -0.394577 \\ \text { H } & 0 & 3.924026 & 0.140091 & -0.159439 \\ \text { H } & 0 & 3.143967 & -1.407944 & 0.170975 \\ \text { H } & 0 & 2.850922 & -0.582200 & -1.381485\end{array}$

$\mathrm{HF}=-793.0154443$

Zero-point correction $=\quad .225303$ (Hartree/Particle)

$6 \mathrm{C}_{2}$

\begin{tabular}{|c|c|c|c|}
\hline & -2.922700 & 0.034393 & -0.622849 \\
\hline & -2.120316 & -1.099167 & -0.577805 \\
\hline & -0.500076 & 0.064529 & -0.056839 \\
\hline & -0.911285 & 0.688278 & 1.016434 \\
\hline & -2.048767 & 1.662460 & 0.880707 \\
\hline & -3.230042 & 0.682602 & 0.446289 \\
\hline & 0.622498 & -0.929886 & 0.107590 \\
\hline & 2.348206 & -0.092621 & 0.063171 \\
\hline & 0.521835 & -1.788712 & 1.375305 \\
\hline & 2.213226 & 1.235153 & 1.363083 \\
\hline & 2.255463 & 0.951736 & -1.483551 \\
\hline & -3.124527 & 0.515671 & -1.592331 \\
\hline & -1.897781 & -1.626526 & -1.502426 \\
\hline & -2.116714 & -1.684250 & 0.335068 \\
\hline & -0.460312 & 0.658447 & -0.974777 \\
\hline & -2.334824 & 2.123520 & 1.947128 \\
\hline & -1.841248 & 2.435654 & -0.031433 \\
\hline & 0.642715 & -1.574493 & -0.779907 \\
\hline & 1.402695 & -2.430878 & 1.471008 \\
\hline & 0.446884 & -1.162430 & 2.267944 \\
\hline & -0.361771 & -2.435654 & 1.351532 \\
\hline & 3.128113 & 1.836900 & 1.322464 \\
\hline & 1.346085 & 1.889481 & 1.236298 \\
\hline & 2.162933 & 0.778015 & 2.356003 \\
\hline
\end{tabular}


Journal of the American Chemical Society

$\begin{array}{lllll}\mathrm{H} & 0 & 3.230057 & 1.432678 & -1.620789 \\ \mathrm{H} & 0 & 2.083725 & 0.311462 & -2.356003 \\ \mathrm{H} & 0 & 1.491272 & 1.736069 & -1.458969\end{array}$

$\mathrm{HF}=-793.0179523$

Zero-point correction=

.225403 (Hartree/Particle)

\begin{tabular}{|c|c|c|c|}
\hline $6 C_{3}$ & & & \\
\hline C & -3.571320 & 0.661346 & 0.79348 \\
\hline C & -2.612289 & 1.573669 & 0.381241 \\
\hline $\mathrm{C}$ & -1.158646 & 0.098938 & 0.650406 \\
\hline 0 & -1.590012 & -0.893021 & -0.07649 \\
\hline B & -2.895676 & -1.551773 & 0.30654 \\
\hline $\mathrm{O}$ & -3.897400 & -0.347137 & 0.05845 \\
\hline C & 0.122498 & 0.770798 & 0.20277 \\
\hline$P$ & 1.527832 & -0.250549 & 1.02792 \\
\hline C & 0.225891 & 0.970581 & -1.31134 \\
\hline C & 2.995605 & 0.821655 & 0.60395 \\
\hline C & 1.760376 & -1.636795 & -0.19497 \\
\hline $\mathrm{H}$ & -3.900681 & 0.660156 & 1.84350 \\
\hline $\mathrm{H}$ & -2.372070 & 2.419800 & 1.02030 \\
\hline $\mathrm{H}$ & -2.439621 & 1.690735 & -0.68281 \\
\hline $\mathrm{H}$ & -1.268921 & -0.001053 & 1.73497 \\
\hline $\mathrm{H}$ & -3.163086 & -2.419815 & -0.47244 \\
\hline $\mathrm{H}$ & -2.899796 & -1.828217 & 1.48692 \\
\hline $\mathrm{H}$ & 0.193558 & 1.734360 & 0.72358 \\
\hline $\mathrm{H}$ & 1.218231 & 1.332947 & -1.59507 \\
\hline $\mathrm{H}$ & 0.029587 & 0.037277 & -1.84352 \\
\hline $\mathrm{H}$ & -0.500244 & 1.712296 & -1.66389 \\
\hline $\mathrm{H}$ & 3.900696 & 0.312225 & 0.95257 \\
\hline $\mathrm{H}$ & 3.106018 & 1.022781 & -0.46746 \\
\hline $\mathrm{H}$ & 2.926636 & 1.773987 & 1.14047 \\
\hline $\mathrm{H}$ & 2.565384 & -2.285507 & 0.16668 \\
\hline $\mathrm{H}$ & 0.843124 & -2.231003 & -0.24494 \\
\hline $\mathrm{H}$ & 2.014969 & -1.292068 & -1.20282 \\
\hline
\end{tabular}

$\mathrm{HF}=-793.0167105$

Zero-point correction $=\quad .225529$ (Hartree/Particle)

Coordinates for transition state geometries for the reaction of the $Z$ propanal enolborane (8) with 2methoxypropanal (3)

\begin{tabular}{llrrr}
\multicolumn{2}{l}{-3B $_{1}$} & & & \\
C & 0 & -2.244339 & 0.619919 & 0.803345 \\
C & 0 & -1.247375 & 1.286255 & 0.100143 \\
C & 0 & -0.331024 & -0.633865 & 0.177094 \\
O & 0 & -1.181427 & -1.444626 & -0.386749 \\
B & 0 & -2.514404 & -1.650116 & 0.313690 \\
O & 0 & -3.074203 & -0.180328 & 0.219406 \\
C & 0 & 0.951157 & -0.393509 & -0.615173 \\
O & 0 & 1.684647 & 0.732376 & -0.150162 \\
C & 0 & 1.761780 & -1.698853 & -0.667145 \\
C & 0 & 2.408051 & 0.586914 & 1.062729 \\
C & 0 & -1.361969 & 1.588500 & -1.371536 \\
H & 0 & -2.185181 & 0.582031 & 1.900681
\end{tabular}


Journal of the American Chemical Society

$\begin{array}{rrrrr}\text { H } & 0 & -0.585617 & 1.922592 & 0.683472 \\ \text { H } & 0 & -0.208633 & -0.720642 & 1.262650 \\ \text { H } & 0 & -3.216110 & -2.370483 & -0.334961 \\ \text { H } & 0 & -2.334427 & -1.935394 & 1.480164 \\ \text { H } & 0 & 0.665161 & -0.133957 & -1.637711 \\ \text { H } & 0 & 2.697784 & -1.526321 & -1.208664 \\ \text { H } & 0 & 1.998947 & -2.084671 & 0.329926 \\ \text { H } & 0 & 1.182175 & -2.461670 & -1.193115 \\ \text { H } & 0 & 2.850021 & 1.565323 & 1.266586 \\ \text { H } & 0 & 1.767487 & 0.311279 & 1.912857 \\ \text { H } & 0 & 3.216125 & -0.151306 & 0.979431 \\ \text { H } & 0 & -0.384308 & 1.829300 & -1.798126 \\ \text { H } & 0 & -1.804764 & 0.748779 & -1.912842 \\ \text { H } & 0 & -2.009460 & 2.461685 & -1.521927\end{array}$

$\mathrm{HF}=-526.2664577$

Zero-point correction $=\quad .220700$ (Hartree/Particle)

\section{$Z-3 B_{2}$}

C 0

$-2.430634$

C $0 \quad-1.282639$

C $0 \quad-0.583206$

O $00-1.412903$

B $0 \quad-2.854050$

O $0 \quad-3.240875$

C $0 \quad 0.827515$

O $0 \quad 1.502213$

C $0 \quad 1.486465$

$\begin{array}{lll}\text { C } & 0 & 2.765228\end{array}$

C $0 \quad-1.151062$

H $0 \quad-2.537613$

H $0 \quad-0.649643$

H $0 \quad-0.624710$

H $0 \quad-3.514908$

H $00-2.882904$

H $0 \quad 0.778717$

H $0 \quad 2.493729$

$\mathrm{H} 0 \quad 1.559631$

H $0 \quad 0.892029$

H $0 \quad 3.095856$

H $0 \quad 3.514923$

H $0 \quad 2.696762$

H $0 \quad-0.104645$

H $0 \quad-1.577484$

H $0 \quad-1.690704$

$\mathrm{HF}=-526.2701336$

Zero-point correction=

$$
\begin{array}{rr}
0.643906 & 0.756378 \\
1.263855 & 0.280121 \\
-0.757385 & 0.259155 \\
-1.395950 & -0.512466 \\
-1.514114 & -0.035736 \\
0.010483 & -0.028366 \\
-0.598373 & -0.282730 \\
0.306183 & 0.578522 \\
-1.987762 & -0.323105 \\
0.743103 & 0.110184 \\
1.718277 & -1.149948 \\
0.483017 & 1.838455 \\
1.748611 & 1.018997 \\
-0.956207 & 1.335754 \\
-2.074936 & -0.861115 \\
-1.942184 & 1.099579 \\
-0.195389 & -1.305069 \\
-1.927719 & -0.747253 \\
-2.405624 & 0.686691 \\
-2.663162 & -0.944077 \\
1.529100 & 0.793594 \\
-0.059937 & 0.110413 \\
1.159317 & -0.906860 \\
1.900238 & -1.411285 \\
0.984558 & -1.838440 \\
2.663177 & -1.292831
\end{array}
$$

\section{.220287 (Hartree/Particle)}
Z-3C'
C 0
2.724487
$-0.225555$
$-0.758286$
C 0
2.012589
$-1.328674$
$-0.291595$
C 0
0.442734
0.104523
$-0.133072$
O 0
0.916183
1.012054
0.669281
B 0
2. 081619
1.836731
0.169983 
Journal of the American Chemical Society

Supporting Information Page 31

$\begin{array}{rrrrr}\mathrm{O} & 0 & 3.165115 & 0.682343 & 0.042465 \\ \mathrm{C} & 0 & -0.722961 & -0.730576 & 0.412338 \\ \mathrm{O} & 0 & -1.771225 & 0.133377 & 0.846802 \\ \mathrm{C} & 0 & -1.221588 & -1.789886 & -0.574707 \\ \mathrm{C} & 0 & 2.186523 & -1.851532 & 1.113083 \\ \mathrm{C} & 0 & -2.308945 & 1.041580 & -0.103821 \\ \mathrm{H} & 0 & 2.712158 & -0.001740 & -1.835022 \\ \mathrm{H} & 0 & 1.697174 & -2.039597 & -1.053833 \\ \mathrm{H} & 0 & 0.363083 & 0.377487 & -1.192459 \\ \mathrm{H} & 0 & 2.448196 & 2.602951 & 1.011551 \\ \mathrm{H} & 0 & 1.846909 & 2.274643 & -0.937241 \\ \mathrm{H} & 0 & -0.400879 & -1.208466 & 1.340424 \\ \mathrm{H} & 0 & -2.153748 & -2.216232 & -0.192932 \\ \mathrm{H} & 0 & -0.498642 & -2.602936 & -0.689835 \\ \mathrm{H} & 0 & -1.417831 & -1.372681 & -1.568726 \\ \mathrm{H} & 0 & 1.387070 & -2.546951 & 1.386505 \\ \mathrm{H} & 0 & 3.133987 & -2.399185 & 1.192444 \\ \mathrm{H} & 0 & 2.213470 & -1.031906 & 1.835007 \\ \mathrm{H} & 0 & -3.165131 & 1.511169 & 0.386200 \\ \mathrm{H} & 0 & -1.590530 & 1.826218 & -0.375381 \\ \mathrm{H} & 0 & -2.661530 & 0.541138 & -1.016907\end{array}$

$\mathrm{HF}=-526.2612998$

Zero-point correction $=\quad .220432$ (Hartree/Particle)

$Z-3 C^{\prime}{ }_{3}$

C $0 \quad-2.793991$

C $0 \quad-2.006378$

C $0 \quad-0.444702$

$\begin{array}{lll}\mathrm{O} & 0 & -0.808014\end{array}$

B $0 \quad-2.033325$

O $0 \quad-3.124100$

C $0 \quad 0.815308$

O $0 \quad 1.913544$

C $0 \quad 1.072342$

C $0 \quad-1.973602$

C $0 \quad 2.202133$

H $0 \quad-2.940170$

H $0 \quad-1.795151$

H $0 \quad-0.488724$

H $0 \quad-2.277573$

H $0 \quad-1.954498$

H $0 \quad 0.765366$

H $0 \quad 2.078125$

H $0 \quad 0.357132$

H $0 \quad 1.026443$

H $0 \quad-1.103226$

H $0 \quad-2.867279$

H $0 \quad-1.962800$

H $0 \quad 3.124115$

H $0 \quad 2.366226$

H $0 \quad 1.402664$

$\mathrm{HF}=-526.268225$

Zero-point correction=

$\begin{array}{rr}-0.310638 & 0.753296 \\ -1.321106 & 0.205292 \\ 0.064545 & 0.517029 \\ 1.106506 & -0.182205 \\ 1.864563 & 0.283157 \\ 0.732025 & 0.070938 \\ -0.655746 & 0.028992 \\ 0.179337 & 0.389282 \\ -2.001373 & 0.697144 \\ -1.586273 & -1.280960 \\ 1.246780 & -0.506577 \\ -0.281525 & 1.842834 \\ -2.160156 & 0.866074 \\ 0.160553 & 1.608215 \\ 2.763763 & -0.467575 \\ 2.115936 & 1.466431 \\ -0.758011 & -1.063995 \\ -2.337540 & 0.431534 \\ -2.763748 & 0.378403 \\ -1.906784 & 1.787704 \\ -2.185791 & -1.565247 \\ -2.148392 & -1.579712 \\ -0.649948 & -1.842850 \\ 1.705978 & -0.14113 \\ 0.870834 & -1.527588 \\ 1.994843 & -0.528946\end{array}$

.220249 (Hartree/Particle) 


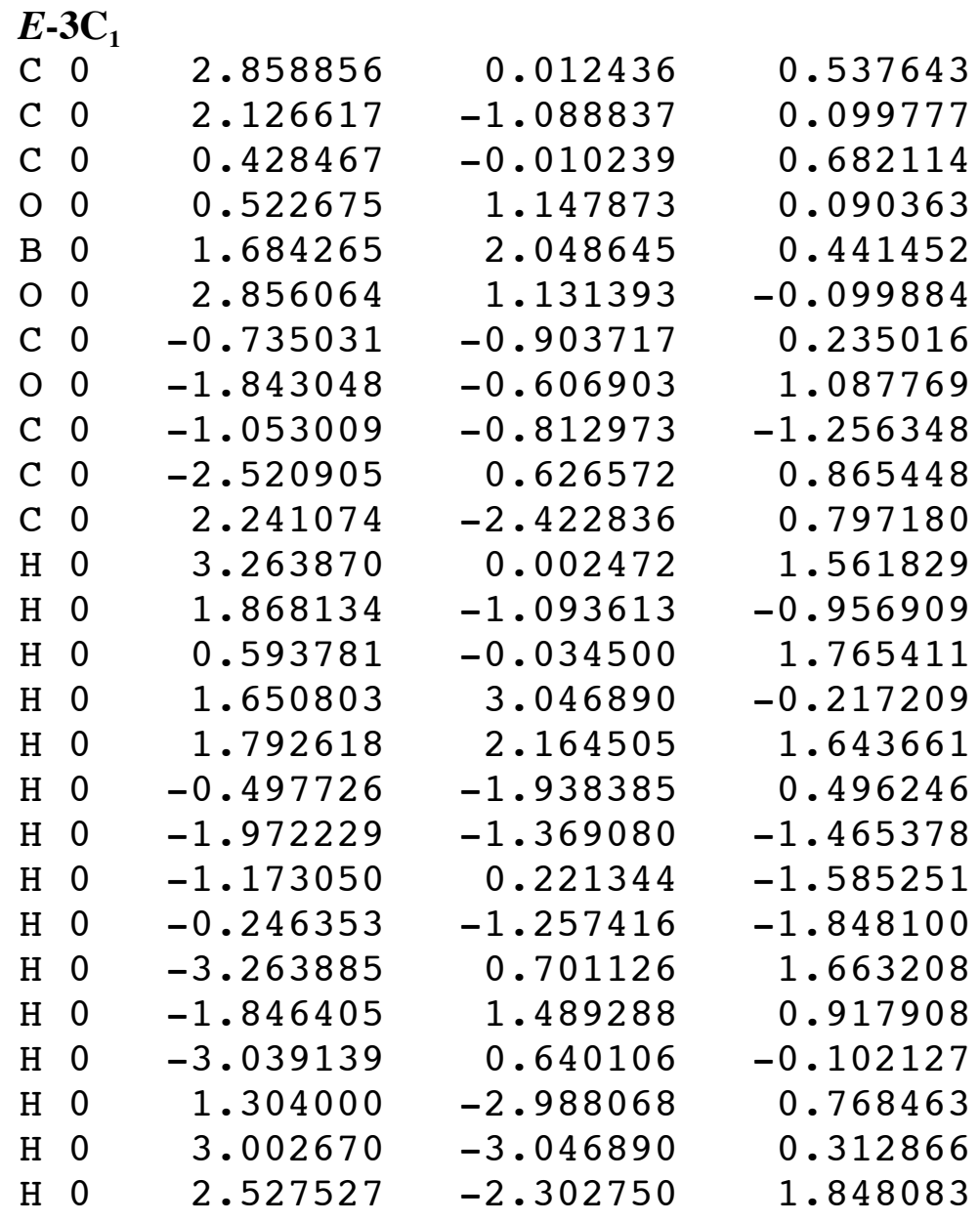

$\mathrm{HF}=-526.2674079$

Zero-point correction=

.220160 (Hartree/Particle)

\begin{tabular}{rlrrr}
\multicolumn{2}{l}{$\boldsymbol{E}-3 C_{2}$} & & & \\
$\mathrm{C}$ & 0 & 3.026230 & -0.259140 & 0.523743 \\
$\mathrm{C}$ & 0 & 1.973160 & -0.939804 & -0.088394 \\
$\mathrm{C}$ & 0 & 0.717834 & 0.530700 & 0.686142 \\
$\mathrm{O}$ & 0 & 1.242203 & 1.668564 & 0.335648 \\
$\mathrm{~B}$ & 0 & 2.608093 & 2.034012 & 0.844818 \\
$\mathrm{O}$ & 0 & 3.436050 & 0.884796 & 0.106827 \\
$\mathrm{C}$ & 0 & -0.633362 & 0.194107 & 0.047806 \\
$\mathrm{O}$ & 0 & -1.580627 & 0.670547 & 1.003662 \\
$\mathrm{C}$ & 0 & -0.829514 & 0.837662 & -1.323166 \\
$\mathrm{C}$ & 0 & -2.841507 & 0.029755 & 0.949844 \\
$\mathrm{C}$ & 0 & 1.599457 & -2.331833 & 0.362183 \\
$\mathrm{H}$ & 0 & 3.358276 & -0.595825 & 1.518921 \\
$\mathrm{H}$ & 0 & 1.779022 & -0.677597 & -1.126572 \\
$\mathrm{H}$ & 0 & 0.778580 & 0.248169 & 1.742691 \\
$\mathrm{H}$ & 0 & 2.957336 & 3.086578 & 0.397079 \\
$\mathrm{H}$ & 0 & 2.693237 & 1.866500 & 2.041977 \\
$\mathrm{H}$ & 0 & -0.733139 & -0.898376 & -0.027969 \\
$\mathrm{H}$ & 0 & -1.821609 & 0.594513 & -1.716064 \\
$\mathrm{H}$ & 0 & -0.731995 & 1.922104 & -1.243958 \\
$\mathrm{H}$ & 0 & -0.086288 & 0.476730 & -2.041977 \\
$\mathrm{H}$ & 0 & -3.436050 & 0.440704 & 1.769424
\end{tabular}


Journal of the American Chemical Society

$\begin{array}{rrrrr}\mathrm{H} & 0 & -3.368454 & 0.218994 & 0.003525 \\ \mathrm{H} & 0 & -2.748184 & -1.059600 & 1.086746 \\ \mathrm{H} & 0 & 0.522095 & -2.516510 & 0.293777 \\ \mathrm{H} & 0 & 2.090912 & -3.086594 & -0.264313 \\ \mathrm{H} & 0 & 1.903000 & -2.510162 & 1.399963\end{array}$

$\mathrm{HF}=-526.2662124$

Zero-point correction $=\quad .219638$ (Hartree/Particle)

$E-3 C_{3}$

C 0

2.773071

1.770233

C 0

C 0

$\begin{array}{ll}\mathrm{O} & 0\end{array}$

B 0

O 0

C 0

$\begin{array}{ll}0 & 0\end{array}$

C 0

C 0

C 0

$\mathrm{H} \quad 0$

$\mathrm{H} \quad \mathrm{O}$

$\mathrm{H} \quad \mathrm{O}$

$\mathrm{H} \quad \mathrm{O}$

$\mathrm{H} \quad \mathrm{O}$

$\mathrm{H} \quad \mathrm{O}$

$\mathrm{H}$

$\mathrm{H}$

$\mathrm{H}$

$\mathrm{H}$

$\mathrm{H}$

$\mathrm{H} \quad 0 \quad-1.684402$

$\mathrm{H} \quad 0 \quad 0.255295$

$\mathrm{H} \quad 0 \quad 1.858368$

$\mathrm{H} 0 \quad 1.521606$

$\mathrm{HF}=-526.2666883$

Zero-point correction=

$$
\begin{array}{rr}
-0.311691 & 0.532867 \\
-0.908508 & -0.231369 \\
0.532013 & 0.539978 \\
1.677963 & 0.326004 \\
1.963425 & 0.992371 \\
0.850311 & 0.255722 \\
0.287231 & -0.240067 \\
0.755386 & 0.578064 \\
1.049179 & -1.557144 \\
-0.202652 & 1.477432 \\
-2.321228 & 0.061783 \\
-0.741013 & 1.521652 \\
-0.555679 & -1.256805 \\
0.174454 & 1.576263 \\
3.036224 & 0.665878 \\
1.704102 & 2.176285 \\
-0.788864 & -0.417786 \\
0.837280 & -2.055084 \\
2.122559 & -1.371994 \\
0.758148 & -2.221802 \\
0.283569 & 2.003159 \\
-1.086304 & 0.944412 \\
-0.538361 & 2.221786 \\
-2.468628 & -0.125198 \\
-3.036224 & -0.576309 \\
-2.594208 & 1.104752
\end{array}
$$

\section{$E-3 A^{\prime}$}

$\begin{array}{rrrrr}\mathrm{C} & 0 & -2.237747 & 1.048798 & 0.350235 \\ \mathrm{C} & 0 & -1.816895 & -0.268539 & 0.432236 \\ \mathrm{C} & 0 & 0.154556 & 0.580521 & 0.610077 \\ \mathrm{O} & 0 & 0.277237 & 1.381287 & -0.400818 \\ \mathrm{~B} & 0 & -0.671631 & 2.570648 & -0.501022 \\ \mathrm{O} & 0 & -2.006012 & 1.787720 & -0.688492 \\ \mathrm{C} & 0 & 1.004227 & -0.688812 & 0.605072 \\ \mathrm{O} & 0 & 0.808426 & -1.518234 & -0.525726 \\ \mathrm{C} & 0 & 2.471451 & -0.296448 & 0.859924 \\ \mathrm{C} & 0 & -2.181534 & -1.139053 & 1.607254 \\ \mathrm{C} & 0 & 1.425079 & -1.138977 & -1.756744 \\ \mathrm{H} & 0 & -2.597290 & 1.549133 & 1.262375 \\ \mathrm{H} & 0 & -1.544922 & -0.766602 & -0.494293\end{array}$


Journal of the American Chemical Society

Supporting Information Page 34

$\begin{array}{rrrrr}\mathrm{H} & 0 & 0.023544 & 1.034714 & 1.601425 \\ \mathrm{H} & 0 & -0.436859 & 3.193726 & -1.495712 \\ \mathrm{H} & 0 & -0.686050 & 3.174103 & 0.552841 \\ \mathrm{H} & 0 & 0.672485 & -1.302322 & 1.449387 \\ \mathrm{H} & 0 & 3.094360 & -1.196671 & 0.861832 \\ \mathrm{H} & 0 & 2.568726 & 0.189621 & 1.836563 \\ \mathrm{H} & 0 & 2.841583 & 0.397400 & 0.100647 \\ \mathrm{H} & 0 & -1.384200 & -1.848511 & 1.852127 \\ \mathrm{H} & 0 & -2.399902 & -0.545151 & 2.502319 \\ \mathrm{H} & 0 & -3.072861 & -1.737518 & 1.379089 \\ \mathrm{H} & 0 & 1.104523 & -1.889572 & -2.483444 \\ \mathrm{H} & 0 & 1.106200 & -0.147659 & -2.088989 \\ \mathrm{H} & 0 & 2.520645 & -1.167282 & -1.688858\end{array}$

$\mathrm{HF}=-526.2678367$

Zero-point correction $=\quad .220243$ (Hartree/Particle)

$E-3 \mathbf{A}^{\prime}$

$\begin{array}{rrrrr}\mathrm{C} & 0 & 2.689148 & -0.198868 & 0.020203 \\ \mathrm{C} & 0 & 1.487411 & -0.881836 & 0.129852 \\ \mathrm{C} & 0 & 0.595947 & 0.934677 & -0.591522 \\ \mathrm{O} & 0 & 1.026459 & 1.856918 & 0.204315 \\ \mathrm{~B} & 0 & 2.518326 & 2.138657 & 0.229294 \\ \mathrm{O} & 0 & 3.008987 & 0.771484 & 0.813507 \\ \mathrm{C} & 0 & -0.883652 & 0.593597 & -0.504974 \\ \mathrm{O} & 0 & -1.206589 & 0.297531 & 0.839798 \\ \mathrm{C} & 0 & -1.678207 & 1.792053 & -1.057312 \\ \mathrm{C} & 0 & 1.180756 & -2.072266 & -0.742767 \\ \mathrm{C} & 0 & -2.485382 & -0.279343 & 1.028778 \\ \mathrm{H} & 0 & 3.300537 & -0.343369 & -0.884430 \\ \mathrm{H} & 0 & 0.949127 & -0.789978 & 1.069200 \\ \mathrm{H} & 0 & 1.005554 & 0.906158 & -1.609070 \\ \mathrm{H} & 0 & 2.759689 & 3.007202 & 1.016098 \\ \mathrm{H} & 0 & 2.933884 & 2.272415 & -0.904739 \\ \mathrm{H} & 0 & -1.074081 & -0.285049 & -1.142563 \\ \mathrm{H} & 0 & -2.748764 & 1.566986 & -1.087692 \\ \mathrm{H} & 0 & -1.361084 & 2.034470 & -2.077560 \\ \mathrm{H} & 0 & -1.510895 & 2.664352 & -0.419952 \\ \mathrm{H} & 0 & 0.123016 & -2.113617 & -1.024857 \\ \mathrm{H} & 0 & 1.776382 & -2.065552 & -1.663086 \\ \mathrm{H} & 0 & 1.400406 & -3.007202 & -0.211349 \\ \mathrm{H} & 0 & -2.537247 & -0.580978 & 2.077560 \\ \mathrm{H} & 0 & -3.300537 & 0.429153 & 0.827271 \\ \mathrm{H} & 0 & -2.627563 & -1.168045 & 0.392990 \\ \mathrm{H} & & -1.526 .267621 & & \end{array}$

$\mathrm{HF}=-526.267621$

Zero-point correction=

.219698 (Hartree/Particle) 


\section{Rotational energy profile for 2-methoxyacetaldehyde:}

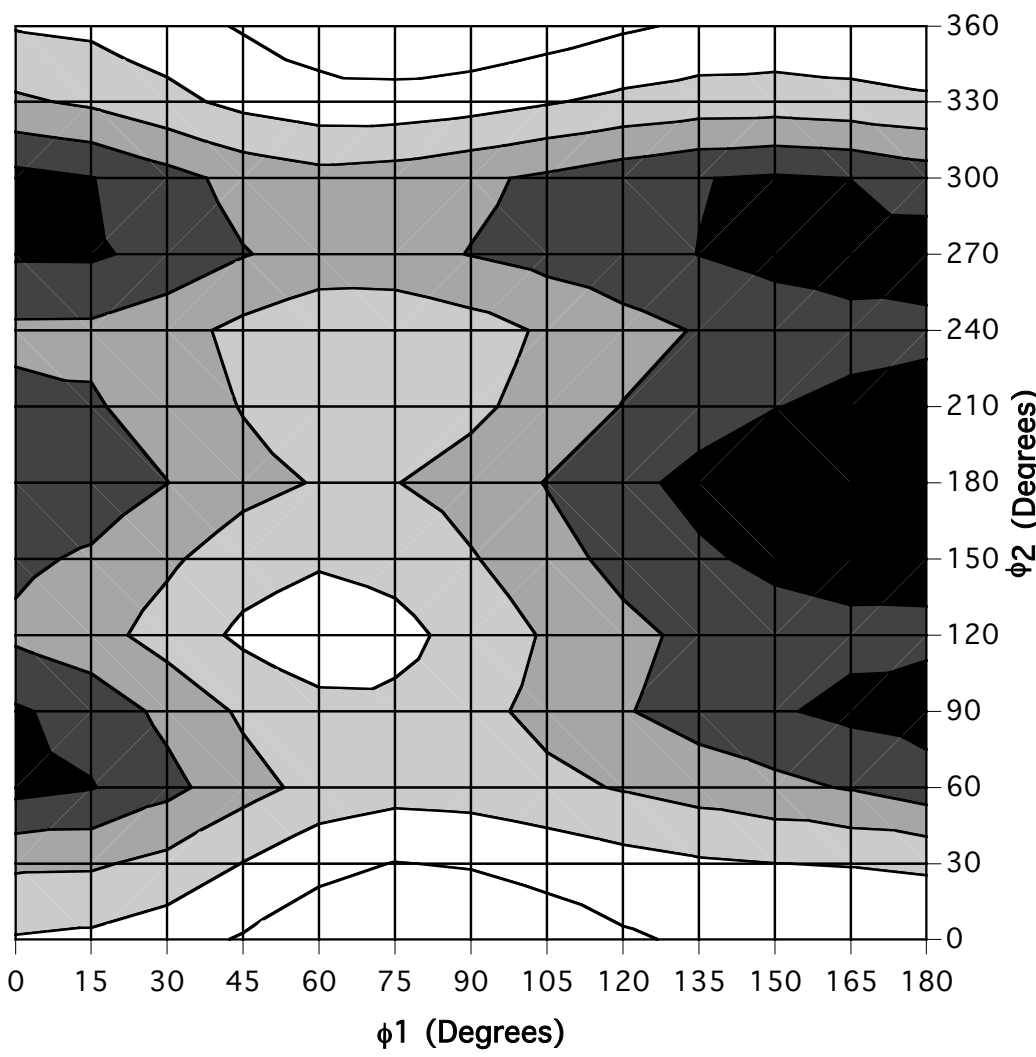

Rel. E

(kcal/mol)

$\square 7.5-9$

$\square 6-7.5$

$\square 4.5-6$

$\square 3-4.5$

$\square 1.5-3$

- 0-1.5

Rotational energy profile for 2-thiomethylacetaldehyde:

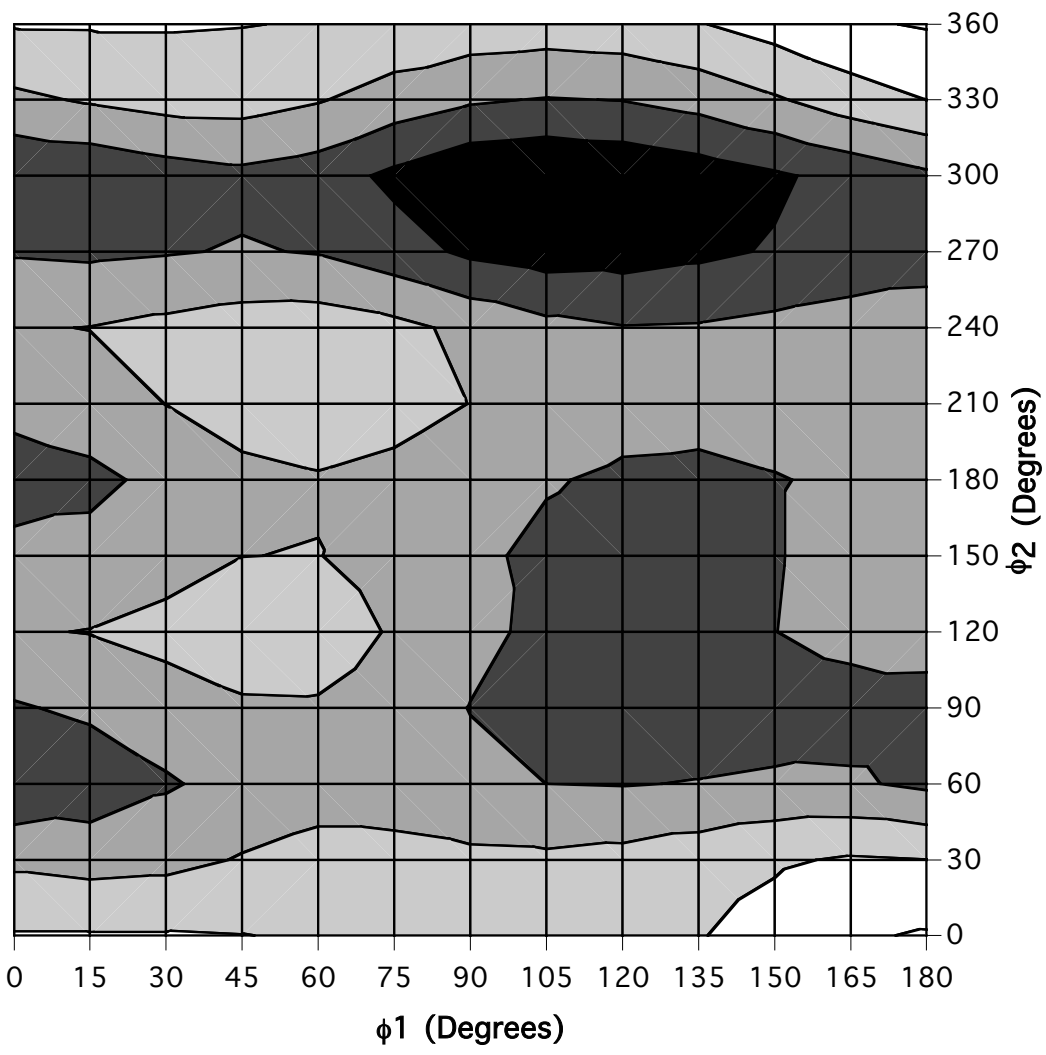

Rel. E

(kcal/mol)

$\square 7.5-9$

$\square 6-7.5$

$\square$ 4.5-6

$\square$ 3-4.5

$\square 1.5-3$

- 0-1.5 
Journal of the American Chemical Society

Rotational energy profile for 2-dimethylaminoacetaldehyde:

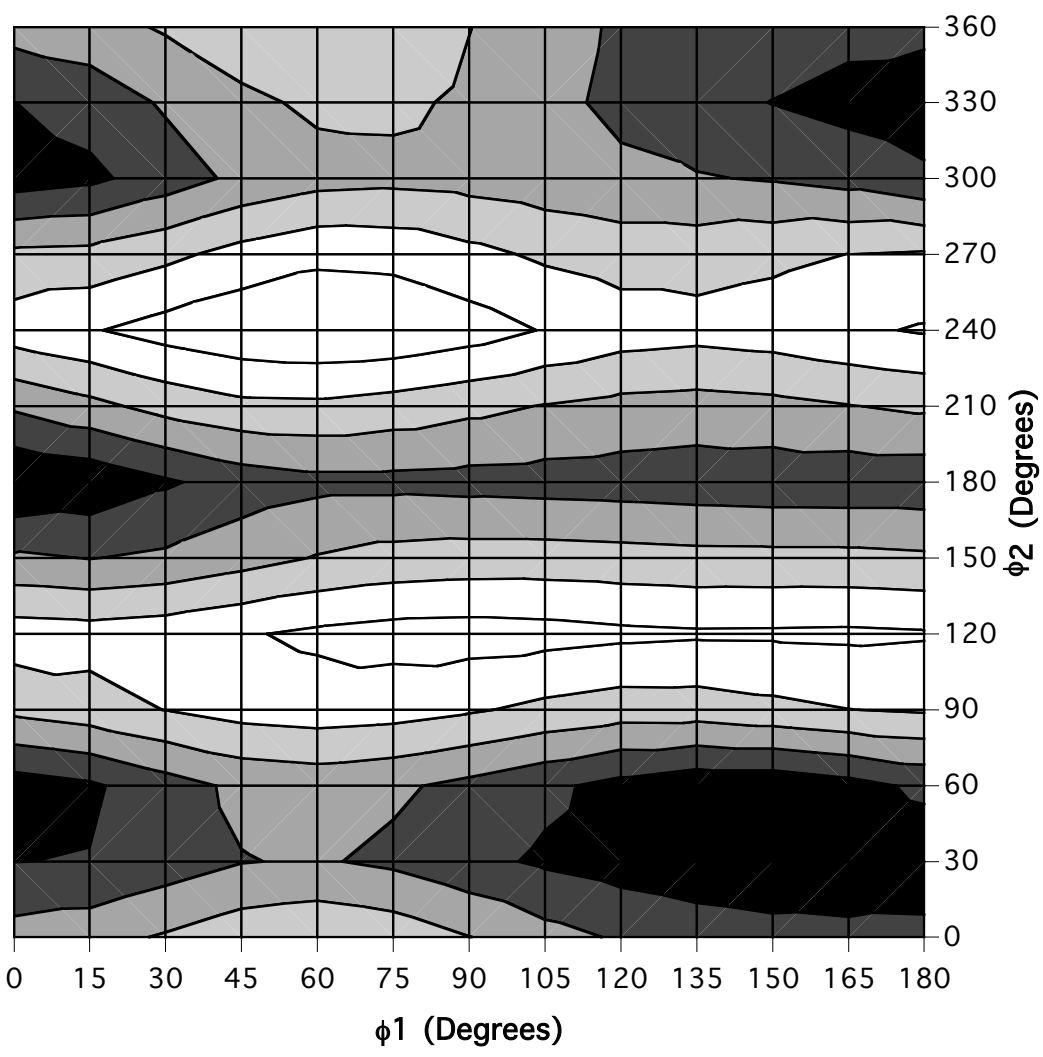

Rotational energy profile for 2-dimethylphosphinoacetaldehyde:

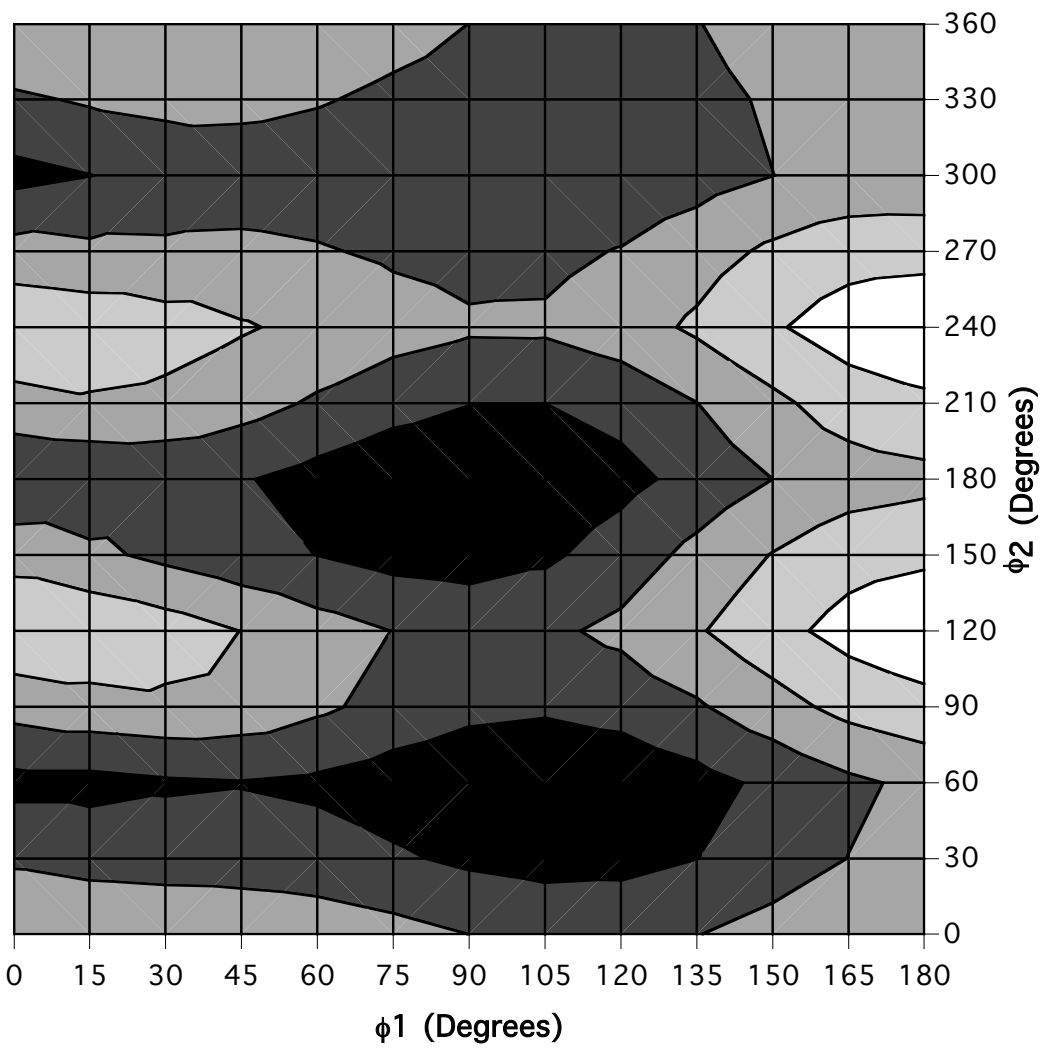

Rel. E (kcal/mol)

\section{$\square 7.5-9$}

$\square 6-7.5$

$\square 4.5-6$

$\square 3-4.5$

$\square 1.5-3$

-0-1.5
Rel. E

(kcal/mol)

$\square 6-7.5$

$\square$ 4.5-6

$\square$ 3-4.5

$\square 1.5-3$

-0-1.5 


\section{Energies for constrained, optimized acetaldehydes}

\begin{tabular}{|c|c|c|c|c|c|}
\hline$\phi_{1}$ & $\phi_{2}$ & $\mathrm{X}=\mathrm{OMe}$ & $\mathrm{X}=\mathrm{SMe}$ & $X=\mathrm{NMe} 2$ & $\mathrm{X}=\mathrm{PMe} 2$ \\
\hline 0 & 0 & -268.3296869 & -591.3182802 & -287.7812973 & -574.4051712 \\
\hline 15 & 0 & -268.3293737 & -591.318275 & -287.7807816 & -574.4052687 \\
\hline 30 & 0 & -268.3284767 & -591.3182798 & -287.7795639 & -574.405468 \\
\hline 45 & 0 & -268.3272531 & -591.318405 & -287.7783476 & -574.4057118 \\
\hline 60 & 0 & -268.3260848 & -591.3186498 & -287.7778085 & -574.4060872 \\
\hline 75 & 0 & -268.3253521 & -591.3189595 & -287.7783603 & -574.4067074 \\
\hline 90 & 0 & -268.3254279 & -591.3194052 & -287.7797721 & -574.4074974 \\
\hline 105 & 0 & -268.3261819 & -591.319617 & -287.7813736 & -574.4080947 \\
\hline 120 & 0 & -268.3271483 & -591.3193327 & -287.782507 & -574.4081521 \\
\hline 135 & 0 & -268.327872 & -591.3185758 & -287.783209 & -574.4075574 \\
\hline 150 & 0 & -268.3281248 & -591.3174749 & -287.7836489 & -574.406577 \\
\hline 165 & 0 & -268.3279647 & -591.3163499 & -287.7838929 & -574.405595 \\
\hline 180 & 0 & -268.3278209 & -591.3158489 & -287.7839681 & -574.4051313 \\
\hline 0 & 30 & -268.3326393 & -591.3213202 & -287.7846307 & -574.4078574 \\
\hline 15 & 30 & -268.3325808 & -591.3217353 & -287.7845218 & -574.4083779 \\
\hline 30 & 30 & -268.3315733 & -591.3214937 & -287.7834608 & -574.4085839 \\
\hline 45 & 30 & -268.3298035 & -591.3206882 & -287.7823132 & -574.4086331 \\
\hline 60 & 30 & -268.3281059 & -591.3200735 & -287.7819664 & -574.408905 \\
\hline 75 & 30 & -268.3274028 & -591.320071 & -287.7827036 & -574.4095336 \\
\hline 90 & 30 & -268.3276552 & -591.3203567 & -287.7839358 & -574.4103171 \\
\hline 105 & 30 & -268.3283179 & -591.3204453 & -287.784965 & -574.4107259 \\
\hline 120 & 30 & -268.3290231 & -591.3201492 & -287.7856868 & -574.4105868 \\
\hline 135 & 30 & -268.3295657 & -591.3195261 & -287.7862877 & -574.4099012 \\
\hline 150 & 30 & -268.3298444 & -591.3187416 & -287.7866518 & -574.4087486 \\
\hline 165 & 30 & -268.3299669 & -591.3181988 & -287.7865388 & -574.4074431 \\
\hline 180 & 30 & -268.3302347 & -591.3184321 & -287.7860802 & -574.4065717 \\
\hline 0 & 60 & -268.3378287 & -591.3254373 & -287.7857536 & -574.4105815 \\
\hline 15 & 60 & -268.3371739 & -591.3247822 & -287.7849646 & -574.410579 \\
\hline 30 & 60 & -268.3353496 & -591.3234945 & -287.783224 & -574.4101671 \\
\hline 45 & 60 & -268.3331468 & -591.322326 & -287.7817017 & -574.4099849 \\
\hline 60 & 60 & -268.3314912 & -591.3218096 & -287.7812696 & -574.4103009 \\
\hline 75 & 60 & -268.3308082 & -591.3220584 & -287.7818092 & -574.4110671 \\
\hline 90 & 60 & -268.330978 & -591.3227069 & -287.7828476 & -574.4117693 \\
\hline 105 & 60 & -268.3316306 & -591.3232289 & -287.7840793 & -574.4120171 \\
\hline 120 & 60 & -268.332442 & -591.3233384 & -287.785374 & -574.4116227 \\
\hline 135 & 60 & -268.333249 & -591.3231181 & -287.7862335 & -574.4106697 \\
\hline 150 & 60 & -268.3339999 & -591.3228528 & -287.7862389 & -574.4093498 \\
\hline 165 & 60 & -268.3348447 & -591.3229403 & -287.7854019 & -574.4079287 \\
\hline 180 & 60 & -268.3359779 & -591.3236658 & -287.7841249 & -574.4069435 \\
\hline 0 & 90 & -268.3373409 & -591.3234526 & -287.7792712 & -574.406604 \\
\hline 15 & 90 & -268.3361196 & -591.3227743 & -287.7784963 & -574.4059635 \\
\hline 30 & 90 & -268.3340661 & -591.3218417 & -287.7773787 & -574.4056091 \\
\hline 45 & 90 & -268.3318925 & -591.3211007 & -287.7765307 & -574.4059892 \\
\hline 60 & 90 & -268.3305073 & -591.321041 & -287.776205 & -574.4070556 \\
\hline 75 & 90 & -268.330449 & -591.3219029 & -287.7764577 & -574.4082908 \\
\hline 90 & 90 & -268.3315043 & -591.3232825 & -287.7771459 & -574.4092115 \\
\hline 105 & 90 & -268.3330027 & -591.3244586 & -287.77805 & -574.4095079 \\
\hline 120 & 90 & -268.334456 & -591.3249731 & -287.778672 & -574.4089757 \\
\hline 135 & 90 & -268.3357252 & -591.3248851 & -287.7786273 & -574.4077628 \\
\hline 150 & 90 & -268.3367848 & -591.3244988 & -287.7780741 & -574.4060338 \\
\hline 165 & 90 & -268.3376069 & -591.3241621 & -287.7774705 & -574.4043416 \\
\hline
\end{tabular}


Journal of the American Chemical Society

$\begin{array}{rrr}180 & 90 & -268.3381184 \\ 0 & 120 & -268.3342007 \\ 15 & 120 & -268.3331534 \\ 30 & 120 & -268.3312886 \\ 45 & 120 & -268.3293909 \\ 60 & 120 & -268.3285001 \\ 75 & 120 & -268.3291248 \\ 90 & 120 & -268.3307485 \\ 105 & 120 & -268.332521 \\ 120 & 120 & -268.3340315 \\ 135 & 120 & -268.3352131 \\ 150 & 120 & -268.3360302 \\ 165 & 120 & -268.336445 \\ 180 & 120 & -268.3364952 \\ 0 & 150 & -268.3351385 \\ 15 & 150 & -268.3343093 \\ 30 & 150 & -268.3326638 \\ 45 & 150 & -268.3309477 \\ 60 & 150 & -268.3301428 \\ 75 & 150 & -268.3306619 \\ 90 & 150 & -268.332073 \\ 105 & 150 & -268.3337532 \\ 120 & 150 & -268.3353487 \\ 135 & 150 & -268.3366707 \\ 150 & 150 & -268.3375692 \\ 165 & 150 & -268.3379683 \\ 180 & 150 & -268.3379426 \\ 0 & 180 & -268.3366389 \\ 15 & 180 & -268.3360993 \\ 30 & 180 & -268.3346859 \\ 45 & 180 & -268.3330659 \\ 60 & 180 & -268.332085 \\ 75 & 180 & -268.3321907 \\ 90 & 180 & -268.3332277 \\ 105 & 180 & -268.3347596 \\ 120 & 180 & -268.3363552 \\ 135 & 180 & -268.3377338 \\ 150 & 180 & -268.338721 \\ 165 & 180 & -268.339266 \\ 180 & 180 & -268.3394319 \\ 0 & 210 & -268.33515 \\ 15 & 210 & -268.3348886 \\ 30 & 210 & -268.3336972 \\ 45 & 210 & -268.3321375 \\ 60 & 210 & -268.3309899 \\ 75 & 210 & -268.3308555 \\ 90 & 210 & -268.3317526 \\ 120 & 210 & -268.3332189 \\ 135 & 210 & -268.3347304 \\ 150 & 210 & -268.3360114 \\ 165 & 210 & -268.3369712 \\ 180 & 210 & -268.3376064 \\ 0 & 240 & -268.3341936 \\ & & \\ 115\end{array}$

$-591.3210105$

$-591.3207688$

$-591.3201695$

$-591.3196284$

$-591.3198235$

$-591.3210389$

$-591.3226429$

$-591.3237566$

$-591.3241033$

$-591.3238632$

$-591.3232531$

$-591.3225375$

$-591.3220997$

$-591.3225168$

$-591.3224582$

$-591.3217032$

$-591.320854$

$-591.3207808$

$-591.3216733$

$-591.3228353$

$-591.323649$

$-591.3239778$

$-591.3238473$

$-591.3233256$

$-591.3226134$

$-591.322104$

$-591.3243448$

$-591.3238123$

$-591.3225638$

$-591.3213942$

$-591.3210036$

$-591.3214559$

$-591.3223028$

$-591.3230759$

$-591.3235427$

$-591.3236148$

$-591.3233245$

$-591.3228907$

$-591.3226798$

$-591.3225134$

$-591.3218544$

$-591.3208062$

$-591.319898$

$-591.3195855$

$-591.3199848$

$-591.3208771$

$-591.3218444$

$-591.3224864$

$-591.3226402$

$-591.3223982$

$-591.3220887$

$-591.3221059$

$-591.3209985$
$-287.7771805$

$-287.7761836$

$-287.7764315$

$-287.7760244$

$-287.7752726$

$-287.774587$

$-287.774109$

$-287.7740042$

$-287.7741951$

$-287.7745473$

$-287.7747579$

$-287.7747211$

$-287.7746298$

$-287.7748289$

$-287.7818478$

$-287.782288$

$-287.7818111$

$-287.7807857$

$-287.7796873$

$-287.7790169$

$-287.7787717$

$-287.7787401$

$-287.778909$

$-287.7791043$

$-287.7791592$

$-287.7791826$

$-287.7794109$

$-287.7869943$

$-287.7863595$

$-287.7849108$

$-287.783531$

$-287.7828776$

$-287.7828754$

$-287.7830447$

$-287.7831931$

$-287.7833439$

$-287.7835801$

$-287.7837221$

$-287.7837874$

$-287.7838055$

$-287.7818478$

$-287.7805209$

$-287.7789753$

$-287.7780055$

$-287.7779297$

$-287.7784401$

$-287.7792$

$-287.7799444$

$-287.7805287$

$-287.7807428$

$-287.7804744$

$-287.7799179$

$-287.7794109$

$-287.7761834$
Supporting Information Page 38

$-574.4034002$

$-574.4031032$

$-574.403177$

$-574.4039045$

$-574.4051272$

$-574.406462$

$-574.4075174$

$-574.4080266$

$-574.4079372$

$-574.4069598$

$-574.4053359$

$-574.4034709$

$-574.4018615$

$-574.4010883$

$-574.40591$

$-574.4069237$

$-574.4080591$

$-574.4090424$

$-574.4099065$

$-574.4107337$

$-574.4110462$

$-574.4103084$

$-574.4087255$

$-574.4068325$

$-574.4050029$

$-574.4035729$

$-574.4030987$

$-574.4098158$

$-574.4096103$

$-574.4093595$

$-574.4096914$

$-574.4106967$

$-574.4117723$

$-574.4122611$

$-574.4119006$

$-574.4106225$

$-574.4090543$

$-574.4074805$

$-574.4062845$

$-574.4057897$

$-574.40591$

$-574.4053706$

$-574.4056766$

$-574.4065737$

$-574.4078013$

$-574.4089604$

$-574.409805$

$-574.4098654$

$-574.4090516$

$-574.4075084$

$-574.4056384$

$-574.4038942$

$-574.4030991$

$-574.4031029$ 


\begin{tabular}{rrr}
15 & 240 & -268.3341562 \\
30 & 240 & -268.3331391 \\
45 & 240 & -268.3316434 \\
60 & 240 & -268.3304828 \\
75 & 240 & -268.3303603 \\
90 & 240 & -268.331272 \\
105 & 240 & -268.3325808 \\
120 & 240 & -268.3337905 \\
135 & 240 & -268.3348156 \\
150 & 240 & -268.3356538 \\
165 & 240 & -268.3362297 \\
180 & 240 & -268.3364976 \\
0 & 270 & -268.3373368 \\
15 & 270 & -268.3373769 \\
30 & 270 & -268.3363088 \\
45 & 270 & -268.334776 \\
60 & 270 & -268.3337817 \\
75 & 270 & -268.3339268 \\
90 & 270 & -268.334726 \\
105 & 270 & -268.3355043 \\
120 & 270 & -268.3362462 \\
135 & 270 & -268.3370739 \\
150 & 270 & -268.3378135 \\
165 & 270 & -268.3382005 \\
180 & 270 & -268.3381109 \\
0 & 300 & -268.3378161 \\
15 & 300 & -268.3371193 \\
30 & 300 & -268.3355184 \\
45 & 300 & -268.3338478 \\
60 & 300 & -268.3330938 \\
75 & 300 & -268.3334215 \\
90 & 300 & -268.3341908 \\
105 & 300 & -268.3350841 \\
120 & 300 & -268.3360835 \\
105 & 360 & -268.3293784 \\
135 & 300 & -268.3369529 \\
150 & 300 & -268.3373619 \\
165 & 300 & -268.337009 \\
135 & 300 & -268.335967 \\
150 & 330 & -268.3326464 \\
165 & 330 & -268.3284823 \\
180 & 330 & -268.3302306 \\
45 & 330 & -268.3318738 \\
60 & 330 & -268.330551 \\
75 & 330 & -268.3292187 \\
10 & -268.328393962 \\
130 & -268.3288856 \\
\hline & & -268.3309566 \\
150 &
\end{tabular}

$-591.3207909$

$-591.3202537$

$-591.3197111$

$-591.3195813$

$-591.3201977$

$-591.3214133$

$-591.3225726$

$-591.3231228$

$-591.323037$

$-591.3226142$

$-591.3221822$

$-591.3220937$

$-591.3234293$

$-591.3236235$

$-591.3233921$

$-591.3230601$

$-591.3233506$

$-591.3245794$

$-591.3260534$

$-591.3267472$

$-591.3265962$

$-591.3260775$

$-591.3254121$

$-591.324702$

$-591.3241918$

$-591.3254766$

$-591.3252108$

$-591.3243497$

$-591.3237967$

$-591.3244219$

$-591.3261533$

$-591.3276479$

$-591.3280053$

$-591.3276008$

$-591.3268997$

$-591.3259612$

$-591.3248068$

$-591.3236587$

$-591.3213269$

$-591.3205903$

$-591.3199359$

$-591.3198728$

$-591.3206692$

$-591.3219223$

$-591.3229521$

$-591.3233614$

$-591.3231577$

$-591.3223818$

$-591.3210963$

$-591.319581$

$-591.3184341$

$-591.3182713$

$-591.3182607$

$-591.3182603$
$-287.7752508$

$-287.7740936$

$-287.7732323$

$-287.7728588$

$-287.7730567$

$-287.7739274$

$-287.7751944$

$-287.7762326$

$-287.7765679$

$-287.7761977$

$-287.7754469$

$-287.774829$

$-287.7792711$

$-287.7791147$

$-287.7780376$

$-287.7765777$

$-287.7755999$

$-287.7757775$

$-287.7767834$

$-287.7778311$

$-287.778457$

$-287.7784571$

$-287.7779748$

$-287.7774019$

$-287.7771804$

$-287.7857546$

$-287.7851332$

$-287.7833994$

$-287.7816496$

$-287.7806554$

$-287.7804513$

$-287.7807243$

$-287.7812193$

$-287.7816927$

$-287.7820475$

$-287.7824125$

$-287.7830599$

$-287.7841249$

$-287.7846307$

$-287.783613$

$-287.7819254$

$-287.7803471$

$-287.7793937$

$-287.7793564$

$-287.7802155$

$-287.7815176$

$-287.782792$

$-287.783816$

$-287.7846565$

$-287.7854108$

$-287.7860803$

$-287.7812973$

$-287.7807816$

$-287.7795639$
$-574.4034922$

$-574.4041006$

$-574.4048814$

$-574.4056942$

$-574.4065224$

$-574.4071428$

$-574.407105$

$-574.4061958$

$-574.4046918$

$-574.4029734$

$-574.4015402$

$-574.4010885$

$-574.406604$

$-574.4069947$

$-574.4070574$

$-574.4069906$

$-574.407287$

$-574.4078374$

$-574.4082446$

$-574.4081095$

$-574.4073482$

$-574.4061028$

$-574.4046626$

$-574.403591$

$-574.4034007$

$-574.4105809$

$-574.4099176$

$-574.4090597$

$-574.4086324$

$-574.4088002$

$-574.4092882$

$-574.4097169$

$-574.4098162$

$-574.4093456$

$-574.4084544$

$-574.4075006$

$-574.4068559$

$-574.4069436$

$-574.4078582$

$-574.4072273$

$-574.4068687$

$-574.4069529$

$-574.4073216$

$-574.4079082$

$-574.4084911$

$-574.4088794$

$-574.4087382$

$-574.4081131$

$-574.4072181$

$-574.4064956$

$-574.4065717$

$-574.4051712$

$-574.4052687$

$-574.405468$ 


$\begin{array}{rrrrrr}45 & 360 & -268.3272572 & -591.3183668 & -287.7783476 & -574.4057118 \\ 60 & 360 & -268.3260911 & -591.3186142 & -287.7778085 & -574.4060872 \\ 75 & 360 & -268.3253555 & -591.3189541 & -287.7783603 & -574.4067074 \\ 90 & 360 & -268.3254324 & -591.3193749 & -287.7797721 & -574.4074974 \\ 105 & 360 & -268.3261839 & -591.3196065 & -287.7813736 & -574.4080947 \\ 120 & 360 & -268.3271498 & -591.3193292 & -287.782507 & -574.4081521 \\ 135 & 360 & -268.3278716 & -591.3185812 & -287.783209 & -574.4075574 \\ 150 & 360 & -268.3281171 & -591.3174831 & -287.7836489 & -574.406577 \\ 165 & 360 & -268.3279587 & -591.3163651 & -287.7838929 & -574.405595 \\ 180 & 360 & -268.3278119 & -591.3158622 & -287.7839681 & -574.4051313\end{array}$

